Geometry $\&$ Topology

Volume 9 (2005) 1915-1951

Published: 17 October 2005

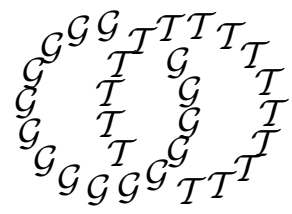

\title{
Representations of polygons of finite groups
}

\author{
Michael Kapovich \\ Department of Mathematics, University of California \\ 1 Shields Ave, Davis, CA 95616, USA \\ Email: kapovich@math.ucdavis.edu
}

\begin{abstract}
We construct discrete and faithful representations into the isometry group of a hyperbolic space of the fundamental groups of acute negatively curved evensided polygons of finite groups.
\end{abstract}

AMS Classification numbers Primary: 20F69

Secondary: 20G05

Keywords: Hyperbolic groups, polygons of groups

Proposed: Benson Farb

Seconded: Martin Bridson, Walter Neumann
Received: 30 January 2004

Revised: 6 October 2005 


\section{Introduction}

There are only few known obstructions for existence of an isometric properly discontinuous action of a Gromov-hyperbolic group $G$ on the real-hyperbolic space $\mathbb{H}^{p}$ for some $p$ :

(1) If $G$ is a group satisfying Kazhdan property (T) then each isometric action $G \curvearrowright \mathbb{H}^{p}$ fixes a point in $\mathbb{H}^{p}$; hence no infinite hyperbolic group satisfying property $(\mathrm{T})$ admits an isometric properly discontinuous action $G \curvearrowright \mathbb{H}^{p}$, for any $p$.

(2) Suppose that $G$ is the fundamental group of a compact Kähler manifold and $G \curvearrowright \mathbb{H}^{p}$ is an isometric properly discontinuous action. Then, according to a theorem of Carlson and Toledo [8], this action factors through an epimorphism $G \rightarrow Q$, where $Q$ is commensurable to a surface group. Hence, unless $G$ itself is commensurable to a surface group, it does not admit an isometric properly discontinuous action $G \curvearrowright \mathbb{H}^{p}$. Examples of Gromov-hyperbolic groups which are Kähler (and are not commensurable to surface groups) are given by the uniform lattices in $P U(m, 1), m \geq 2$, as well as the fundamental groups of compact negatively curved Kähler manifolds (see [20]).

On the positive side, by a theorem of Bonk and Schramm [3], each Gromovhyperbolic group admits a quasi-isometric embedding to a real-hyperbolic space.

The goal of this paper is to find a better "demarcation line" between hyperbolic groups satisfying property $(\mathrm{T})$ and groups acting discretely on real-hyperbolic spaces. In this paper we will show that a large class of 2-dimensional Gromovhyperbolic groups admits isometric properly discontinuous convex-cocompact actions on real-hyperbolic spaces. We consider a 2-dimensional negatively curved acute polygon $\mathcal{P}$ of finite groups (see section 2.2 for more details). Let $G:=\pi_{1}(\mathcal{P})$ be the fundamental group of this polygon, we refer the reader to [7. Chapter II, section 12] for the precise definitions.

Our main result is:

Theorem 1.1 Suppose that $n=2 k$ is even. Then the group $G$ admits a discrete, faithful, convex-cocompact action $\rho$ on a constant curvature hyperbolic space $\mathbb{H}^{p}$, where $p<\infty$ depends on the polygon $\mathcal{P}$.

Our technique in general does not work in the case when $n$ is odd: We were unable to construct a representation. However in section [ 6 we will construct $\rho$ and prove that it is discrete, faithful, convex-cocompact for a special class of odd-sided $n$-gons of groups, provided that $n \geq 5$. 
Conjecture 1.2 The assertion of Theorem 1.1 remains valid for all odd $n \geq 5$.

In contrast, if $\mathcal{P}$ is a triangle of finite groups where the vertex links are connected graphs with the $1^{\text {st }}$ positive eigenvalue of the Laplacian $>1 / 2$, then the group $G=\pi_{1}(\mathcal{P})$ satisfies property $(\mathrm{T})$, see [1]. Hence (provided that $G$ is infinite) the group $G$ cannot act properly discontinuously on $\mathbb{H}^{p}$ for any $p$. Thus, it appears, that (at least for the polygons of finite groups) the "demarcation line" which we are trying to find, is hidden somewhere among quadrilaterals and triangles of groups. We will address this issue in another paper.

Recall that, by a theorem of Dani Wise [24, $G$ is residually finite (actually, Wise proves that $G$ has separable quasi-convex subgroups, which is used in the proof of our main theorem).

Corollary 1.3 The group $G$ is linear.

Remark 1.4 A very different proof of linearity of $G$ was given by Wise and Haglund, who used an embedding of $G$ to a right-angled Coxeter group, 14.

The following problem is open even for right-angled Coxeter groups of virtual cohomological dimension 2.

Problem 1.5 Suppose that $G$ is a Gromov-hyperbolic Coxeter group. Is $G$ isomorphic to a discrete subgroup of $\operatorname{Isom}\left(\mathbb{H}^{n}\right)$ for some $n$ ? Note that if one insists that the Coxeter generators act on $\mathbb{H}^{n}$ as reflections, then there are examples of Gromov-hyperbolic Coxeter groups which do not admit such actions on $\mathbb{H}^{n}$, see [10.

In section 8, we give an example of a nonlinear Gromov-hyperbolic group.

The proof of the main theorem splits in two parts: (1) Construction of $\rho,(2)$ proof of discreteness. To prove discreteness of $\rho$ we show that there exists a $\rho-$ equivariant quasi-isometric embedding $\mu: X \rightarrow \mathbb{H}^{p}$, where $X$ is the universal cover of the polygon $\mathcal{P}$. This proves that the action $\rho: G \curvearrowright \mathbb{H}^{p}$ is properly discontinuous and convex-cocompact. (A priori this action can have finite kernel. In section 7 we explain how to deal with this issue.) The proof that $\mu$ is a quasi-isometric embedding is based on the following theorem of independent interest: 
Theorem 1.6 Suppose that $X$ is a 2-dimensional regular cell complex, which is equipped with a CAT $(-1)$ path-metric so that each face of $X$ is isometric to a right-angled regular $n$-gon in $\mathbb{H}^{2}$. Let $\mu: X \rightarrow \mathbb{H}^{p}$ is a continuous map which is a (totally-geodesic) isometric embedding on each face of $X$. Assume also that for each pair of faces $F^{\prime}, F^{\prime \prime} \subset X$ which intersect non-trivially a common face $F \subset X$, we have:

$$
\operatorname{Span}\left(\mu\left(F^{\prime}\right)\right) \perp \operatorname{Span}\left(\mu\left(F^{\prime \prime}\right)\right) .
$$

Then $\mu$ is a quasi-isometric embedding.

Our construction of representations $\rho$ was inspired by the paper of Marc Bourdon, 4], where he proves a theorem which is a special case of Theorem 1.1] In his paper Bourdon considers $n$-gons of finite groups where the edge groups are cyclic and the vertex groups are direct products of the adjacent edge groups, under the extra assumption that the orders of the edge groups are much smaller that $n$.

Acknowledgments During the work on this paper I was visiting the Max Plank Institute (Bonn), I was also supported by the NSF grants DMS-02-03045 and DMS-04-05180. I am grateful to Tadeusz Januszkiewicz for inspirational and helpful conversations during the work on this paper. I first tried to prove discreteness of certain representations by verifying that an equivariant map is a quasi-isometric embedding, in a joint project with Bernhard Leeb in 1998. Although our attempt back then was unsuccessful, I am grateful to Bernhard Leeb for that effort. I am also grateful to the referees of this paper for numerous suggestions and to Mark Sapir for discussions of Theorem 8.1 .

\section{Preliminaries}

Notation If $\Sigma$ is a (finite) set, we define a Euclidean vector space $V e c t(S)$ to be the vector space $L_{2}(S)$, where $S$ forms an orthonormal basis (we identify each 1-point subset of $S$ with its characteristic function in $\left.L_{2}(S)\right)$. Suppose that $S \subset \mathbb{H}^{q}$. Then $\operatorname{Span}(E)$ will denote the smallest totally-geodesic subspace in $\mathbb{H}^{q}$ which contains $S$. If $E \subset \mathbb{H}^{q}$ is a geodesic segment, then $\operatorname{Bis}(E)$ will denote the perpendicular bisector of $E$.

Suppose that $E_{1}, E_{2} \subset E$ are subspaces of a Euclidean vector space $E$, whose intersection is $E_{3}$. We say that $E_{1}, E_{2}$ intersect orthogonally if $E_{1} / E_{3}, E_{2} / E_{3}$ are contained in the orthogonal complements of each other in the Euclidean space $E / E_{3}$. 
Suppose that $H^{\prime}, H^{\prime \prime}$ are totally-geodesic subspaces in $\mathbb{H}^{p}$. We say that $H^{\prime}, H^{\prime \prime}$ intersect orthogonally if

$$
H^{\prime} \backslash H^{\prime \prime} \neq \emptyset, H^{\prime \prime} \backslash H^{\prime} \neq \emptyset
$$

and for some (equivalently, for every) point $x \in H^{\prime} \cap H^{\prime \prime}$ we have:

$$
T_{x}\left(H^{\prime}\right), T_{x}\left(H^{\prime \prime}\right) \subset T_{x}\left(\mathbb{H}^{p}\right) \text { intersect orthogonally. }
$$

Totally geodesic subspaces $H^{\prime}, H^{\prime \prime} \subset \mathbb{H}^{p}$ are said to be orthogonal to each other if either:

(a) $H^{\prime}, H^{\prime \prime}$ intersect orthogonally, or

(b) $H^{\prime}, H^{\prime \prime}$ are within positive distance from each other and for the unique shortest geodesic segment $\sigma:=\overline{x^{\prime} x^{\prime \prime}} \subset \mathbb{H}^{p}$ connecting $H^{\prime}$ to $H^{\prime \prime}$, the totallygeodesic subspaces $H^{\prime \prime}, \gamma_{\sigma}\left(H^{\prime}\right)$ intersect orthogonally. Here $\gamma_{\sigma}$ is the hyperbolic translation along $\sigma$ which sends $x^{\prime}$ to $x^{\prime \prime}$.

We will use the notation $H^{\prime} \perp H^{\prime \prime}$ for subspaces $H^{\prime}, H^{\prime \prime}$ orthogonal to each other. Clearly, $H^{\prime} \perp H^{\prime \prime} \Longleftrightarrow H^{\prime \prime} \perp H^{\prime}$.

\subsection{Discrete subgroups of $\operatorname{Isom}\left(\mathbb{H}^{n}\right)$}

Recall that a map $f: X \rightarrow Y$ between two metric spaces is called an $(L, A)$ quasi-isometric embedding if for all $x, x^{\prime} \in X$ we have:

$$
L^{-1} d\left(x, x^{\prime}\right)-A \leq d\left(f(x), f\left(x^{\prime}\right)\right) \leq L d\left(x, x^{\prime}\right)+A,
$$

where $L>0$. An $(L, A)$ quasi-isometry is an $(L, A)$ quasi-isometric embedding $X \stackrel{f}{\rightarrow} Y$ such that each point of $Y$ is within distance $\leq A$ from a point in $\operatorname{Im}(f)$.

A map $f$ is called a quasi-isometry (resp. a quasi-isometric embedding) if it is an $(L, A)$ quasi-isometry (resp. quasi-isometric embedding) for some $L$ and $A$.

An $(L, A)$ quasi-geodesic segment in a metric space $X$ is an $(L, A)$ quasiisometric embedding $f:[0, T] \rightarrow X$, where $[0, T]$ is an interval in $\mathbb{R}$. By abusing notation we will sometimes refer to the image $\operatorname{Im}(f)$ of an $(L, A)$ quasi-geodesic segment $f$ as an $(L, A)$ quasi-geodesic segment. Recall that by the Morse lemma (see for instance [17, Lemma 3.43]), quasi-geodesics in $\mathbb{H}^{n}$ are stable:

There is a function $D=D(L, A)$ such that for each $(L, A)$ quasi-geodesic segment $f:[0, T] \rightarrow \mathbb{H}^{n}$, the Hausdorff distance between $\operatorname{Im}(f)$ and the geodesic segment

$$
\overline{f(0) f(T)} \subset \mathbb{H}^{n}
$$


connecting the end-points of $\operatorname{Im}(f)$ is at most $D$.

We will use the notation $\mathbb{H}^{n}$ for the real-hyperbolic $n$-space; its curvature is normalized to be equal to -1 . The space $\mathbb{H}^{n}$ has a geometric compactification $\overline{\mathbb{H}}^{n}=\mathbb{H}^{n} \cup S^{n-1}$. For a subset $S \subset \mathbb{H}^{n}$ we let $\bar{S}$ denote its closure in $\overline{\mathbb{H}}^{n}$.

Discrete subgroups $G \subset \operatorname{Isom}\left(\mathbb{H}^{n}\right)$ are called Kleinian groups.

The convex hull $C(G)$ of a Kleinian group $G \subset \operatorname{Isom}\left(\mathbb{H}^{n}\right)$ is the smallest nonempty closed convex $G$-invariant subset $C \subset \mathbb{H}^{n}$. The convex hull exists for each $G$ whose limit set has cardinality $\neq 1$. The convex hull is unique unless $G$ is finite.

Definition 2.1 A Kleinian group $G \subset \operatorname{Isom}\left(\mathbb{H}^{n}\right)$ is called convex-cocompact if $C(G)$ exists and the quotient $C(G) / G$ is compact.

Lemma 2.2 Suppose we have a representation $\rho: G \rightarrow \operatorname{Isom}\left(\mathbb{H}^{n}\right)$ of a finitelygenerated group $G$. Then the action $\rho: G \curvearrowright \mathbb{H}^{n}$ is properly discontinuous and convex-cocompact iff there exists a $G$-equivariant quasi-isometric embedding $f: \Gamma_{G} \rightarrow \mathbb{H}^{n}$, where $\Gamma_{G}$ is a Cayley graph of $G$.

Proof First, suppose that $\rho: G \curvearrowright \mathbb{H}^{n}$ properly discontinuous and convexcocompact. Then, because $C(G)$ is a geodesic metric space, there exists a $G$-equivariant quasi-isometry $f: \Gamma_{G} \rightarrow C(G)$. Composing this map with the isometric embedding $\iota: C(G) \rightarrow \mathbb{H}^{n}$, we conclude that $f: \Gamma_{G} \rightarrow \mathbb{H}^{n}$ is a quasiisometric embedding.

Conversely, suppose that $f: \Gamma_{G} \rightarrow \mathbb{H}^{n}$ is an equivariant quasi-isometric embedding. In particular, $f$ is a proper map. Hence, if for $1 \in \Gamma_{G}$ we set $o:=f(1)$, then for each compact subset $K \subset \mathbb{H}^{n}$ there are only finitely many elements $g \in G$ such that $g(o) \in K$. Therefore the action $G \curvearrowright \mathbb{H}^{n}$ is properly discontinuous. In particular, it has finite kernel.

Observe that stability of quasi-geodesics in $\mathbb{H}^{n}$ implies that $\operatorname{Im}(f)$ is quasiconvex, ie, there exists a constant $c<\infty$ such that for any two points $x, y \in$ $\operatorname{Im}(f)$ the geodesic segment $\overline{x y}$ is contained in a $c$-neighborhood $N_{c}(\operatorname{Im}(f))$ of $\operatorname{Im}(f)$. On the other hand, by [6. Proposition 2.5.4], there exists $R=R(c)$ such that the convex hull of each $c$-quasi-convex subset $S \subset \mathbb{H}^{n}$ is contained in the $R$-neighborhood $N_{R}(S)$. Thus, the convex subset $C(\rho(G)) \subset \mathbb{H}^{n}$ is contained in $N_{R(c)}(\operatorname{Im}(f))$. Since $G$ acts cocompactly on $\operatorname{Im}(f)$ it follows that $G$ acts cocompactly on $C(G)$. Therefore $\rho(G)$ is convex-cocompact. 
Remark 2.3 (1) Clearly, instead of $\Gamma_{G}$ in the above lemma one can use any geodesic metric space on which $G$ acts isometrically, properly discontinuously and cocompactly.

(2) The above lemma shows that existence of a $G$-equivariant quasi-isometric embedding $f: \Gamma_{G} \rightarrow \mathbb{H}^{n}$ implies that $\rho$ has finite kernel. However it does not exclude the possibility that this kernel is nontrivial.

Definition 2.4 Let $G$ be a group with a Cayley graph $\Gamma_{G}$. A subgroup $H \subset G$ is called quasi-convex if the orbit $H \cdot 1 \subset \Gamma_{G}$ is quasi-convex, ie, there exists a number $D$ so that each geodesic segment $\sigma \subset \Gamma_{G}$ with vertices in $H \cdot 1$ is contained in $N_{D}(H \cdot 1)$.

If $G$ is Gromov-hyperbolic then quasi-convexity of $H$ is independent of the choice of Cayley graph $\Gamma_{G}$.

\subsection{Geometry of polygons of groups}

Consider an $n$-gon $P(n \geq 5)$ with vertices $x_{i}$ and edges $e_{j}, 1 \leq i, j \leq n$. Throughout we will be working $\bmod n$, ie, $q n+i$ will be identified with $i$ for $i \in\{1, \ldots, n\}$. We will be assuming that each edge $e_{i}$ has the vertices $x_{i}, x_{i+1}$. We will regard $P$ as a (2-dimensional) cell complex and its poset $\operatorname{Pos}(P)$ as a (small) category. A polygon of groups $\mathcal{P}$ based on $P$ is a covariant functor from $\operatorname{Pos}(P)$ to the category of groups and monomorphisms.

In other words, a polygon of groups $\mathcal{P}$ based on $P$ is a collection of groups $G_{x_{i}}, G_{e_{i}}, G_{F}$ assigned to the vertices, edges and the 2-face $F$ of $P$, together with monomorphisms

$$
G_{F} \rightarrow G_{e} \rightarrow G_{x}
$$

for each edge $e$ containing the vertex $x$, so that the following diagrams are commutative:

$$
\begin{array}{ccc}
G_{x} & \leftarrow & G_{e} \\
& \nwarrow & \uparrow \\
& G_{F}
\end{array}
$$

The direct limit of the above diagrams of monomorphisms is the fundamental group $G=\pi(\mathcal{P})$ of the polygon $\mathcal{P}$. If the vertex, edge and face groups of $\mathcal{P}$ embed naturally into $G$, the polygon $\mathcal{P}$ is called developable. Not every polygon of groups is developable, however under a certain nonpositive curvature assumption on $\mathcal{P}$, the polygon $\mathcal{P}$ is developable, see [7]. 
Curvature and angles For each vertex $x_{i} \in P$ define a graph $L k_{x_{i}}$ as follows. The vertices of $L k_{x_{i}}$ are the right cosets $g G_{e_{i}}, g G_{e_{i-1}}, g \in G_{x_{i}}$. The vertices $v, w$ are connected by a (single) edge iff there exists $g \in G_{x_{i}}$ such that $g(\{v, w\})=\left\{G_{e_{i}}, G_{e_{i-1}}\right\}$. Thus the group $G_{x_{i}}$ acts on $L k_{x_{i}}$ with the quotient being the edge connecting $G_{e_{i}}, G_{e_{i-1}}$. We metrize the graph $L k_{x_{i}}$ by assigning the same length $\alpha_{i}$ to each edge, so that the group $G_{x_{i}}$ acts isometrically. Then the angle between the subgroups $G_{e_{i}}, G_{e_{i-1}}$ is the least number $\alpha_{i}$ such that the metric graph $L k_{x_{i}}$ is a CAT(1) space, ie, the length of the shortest embedded cycle in $L k_{x_{i}}$ is at least $2 \pi$. Equivalently, the angle between $G_{e_{i}}, G_{e_{i-1}}$ equals

$$
2 \pi / \operatorname{girth}\left(L k_{x_{i}}\right) \text {. }
$$

We will say that the polygon $\mathcal{P}$ is acute (or has acute angles) if the angle $\alpha_{i}$ between each pair of edge groups $G_{e_{i}}, G_{e_{i-1}}$ is at most $\pi / 2$.

We refer the reader to [7, Chapter II, section 12] for the precise definitions of the nonpositive/negative curvature of $\mathcal{P}$; various examples of negatively curved polygons of groups can be found in [1], 7, Chapter II, section 12] and [22. Instead, we state the following equivalent definition of negative curvature:

There exists a 2-dimensional simply-connected regular cell complex $X$ (the universal cover of $\mathcal{P}$ ) together with a path-metric on $X$ whose restriction to each face of $X$ has constant curvature -1 , so that:

(1) Each face of $X$ is isometric to an $n$-gon in $\mathbb{H}^{2}$ with angles $\alpha_{1}, \ldots, \alpha_{n}$.

(2) Each cell in $X$ is convex.

(3) There exists an isometric cellular action $G \curvearrowright X$ which is transitive on $2-$ cells.

(4) The stabilizer of each 2 -face $F \subset X$ is isomorphic to $G_{F}$, it fixes $F$ pointwise.

(5) The stabilizer of each edge $e$ of $F$ is isomorphic to $G_{e}$ and it fixes $e$ pointwise.

(6) The stabilizer of each vertex $x$ of $F$ is isomorphic to $G_{x}$.

(7) The inclusion maps $G_{F} \hookrightarrow G_{e} \hookrightarrow G_{x}$ coincide with the monomorphisms $G_{F} \rightarrow G_{e} \rightarrow G_{x}$ in the definition of $\mathcal{P}$.

Note that the link in $X$ of each vertex $x_{i} \in F$ is isometric to $L k_{x_{i}}$ (where each edge has the length $\alpha_{i}$ ). Thus the above complex $X$ is a CAT(-1) metric space.

Throughout the paper we will be using only the following corollary of negative curvature for acute polygons of groups: 
Corollary 2.5 If $\mathcal{P}$ is negatively curved then there is a CAT $(-1)$ complex $X$ where each face is isometric to a regular right-angled polygon in $\mathbb{H}^{2}$, so that the properties (2)-(7) are satisfied.

In this paper we will consider only the case when the vertex groups are finite, thus the action $G \curvearrowright X$ is properly discontinuous and cocompact, which implies that $X$ is equivariantly quasi-isometric to a Cayley graph of $G$.

We now return to the original polygon $\mathcal{P}$ assuming that it has even number of sides. Let $o$ denote the center of the face $F$ and let $m_{j}$ be the midpoint of the edge $e_{j} \subset F$. We consider two subgraphs $\Gamma_{\text {even }}, \Gamma_{\text {odd }} \subset F$ which are obtained by conning off from $o$ the sets

$$
m_{\text {even }}:=\left\{m_{2 j}, j=1, \ldots, n / 2\right\}, m_{\text {odd }}:=\left\{m_{2 j-1}, j=1, \ldots, n / 2\right\}
$$

respectively. Let $G_{\text {even }}, G_{\text {odd }}$ denote the subgroups of $G$ generated by the elements of

$$
G_{e_{2 j}}, j=1, \ldots, n / 2
$$

and

$$
G_{e_{2 j-1}}, j=1, \ldots, n / 2
$$

respectively. Define subgraphs $T_{\text {even }}$ and $T_{\text {odd }}$ to be the orbits

$$
G_{\text {even }} \cdot \Gamma_{\text {even }} \text { and } G_{\text {odd }} \cdot \Gamma_{\text {odd }} \text {. }
$$

We define a new path-metric $\tau$ on the complex $X$ by declaring the closure of each component of $X \backslash\left(T_{\text {even }} \cup T_{\text {odd }}\right)$ to be a unit Euclidean square. Clearly, the group $G$ acts on $(X, \tau)$ isometrically and $(X, \tau)$ is a $\operatorname{CAT}(0)$ metric space.

The groups $G_{\text {even }}, G_{\text {odd }}$ act on the graphs $T_{\text {even }}, T_{\text {odd }}$ with the fundamental domains $\Gamma_{\text {even }}, \Gamma_{\text {odd }}$ respectively. It therefore follows that if $g \in G$ and $g\left(T_{\text {even }}\right) \cap T_{\text {even }} \neq \emptyset$ (resp. $\left.g\left(T_{\text {odd }}\right) \cap T_{\text {odd }} \neq \emptyset\right)$ then $g \in G_{\text {even }}$ (resp. $\left.g \in G_{\text {odd }}\right)$.

Lemma 2.6 The subgraphs $T_{\text {even }}, T_{\text {odd }} \subset X$ are convex subsets in $X$ isometric (with respect to the path-metric induced from $(X, \tau)$ ) to a tree.

Proof (1) First, let us prove that $T_{\text {even }}, T_{\text {odd }} \subset X$ are convex. Since $X$ is a CAT(0) space, and $T_{\text {even }}, T_{\text {odd }}$ are connected, it suffices to test convexity at each vertex of $T_{\text {even }}, T_{\text {odd }}$. However, by the definition of the metric $\tau$, the angle between different edges of $T_{\text {even }}\left(\right.$ resp. $\left.T_{\text {odd }}\right)$ at each vertex of $T_{\text {even }}\left(\right.$ resp. $T_{\text {odd }}$ ) is $\geq \pi$. Therefore convexity follows.

(2) Since, $T_{\text {even }}, T_{\text {odd }} \subset(X, \tau)$ are convex, it follows that they are contractible. Therefore these graphs are isometric to metric trees. It is clear that $T_{\text {even }}$ and $T_{\text {odd }}$ are isometric to each other. 
Corollary 2.7 Each subgroup $G_{\text {even }}, G_{\text {odd }}$ is a quasi-convex subgroup of $G$.

Proof Cayley graphs $\Gamma_{G}, \Gamma_{G_{\text {even }}}, \Gamma_{G_{\text {odd }}}$ are quasi-isometric to $(X, \tau), T_{\text {even }}$, $T_{\text {odd }}$ respectively. Recall that $X$ is Gromov-hyperbolic. Therefore, by combining stability of quasi-geodesics in Gromov-hyperbolic geodesic metric spaces and convexity of $T_{\text {even }}, T_{\text {odd }} \subset X$, we conclude that $G_{\text {even }}, G_{\text {odd }}$ are quasi-convex subgroups of $G$.

We define functions odd $(i)$ and even $(i)$ by

$$
\operatorname{odd}(i)=\left\{\begin{array}{c}
i, \text { if } i \text { is odd } \\
i-1, \text { if } i \text { is even }
\end{array} \text { and } \operatorname{even}(i)=\left\{\begin{array}{c}
i, \text { if } i \text { is even } \\
i-1, \text { if } i \text { is odd }
\end{array}\right.\right.
$$

To motivate the following definition, observe that the group $G$ is generated by the elements $g_{l}$ of the vertex subgroups $G_{x_{l}}$. It will be very important for the later analysis to find out which products of pairs of generators $f=h_{j}^{-1} g_{i}, h_{j} \in$ $G_{x_{j}}, g_{i} \in G_{x_{i}}$, preserve the trees $T_{o d d}, T_{\text {even }}$. Note that the answer is clear for some of these products:

(a) If $g_{i}$ preserves $e_{\text {odd(i) }}$ (resp. $\left.e_{\text {even }(i)}\right)$ and $h_{j}^{-1}$ preserves $e_{\text {odd(j) }}$ (resp. $\left.e_{\text {even }(j)}\right)$, then $g_{i}, h_{j}^{-1} \in G_{\text {odd }}$ (resp. $G_{\text {even }}$ ) and hence $f$ also preserves $T_{\text {odd }}$ (resp. $T_{\text {even }}$ ).

(b) If $i=j$ and the product $f=h_{i}^{-1} g_{i}$ preserves the edge $e_{o d d(i)}$ (resp. $\left.e_{\text {even }(i)}\right)$, then $f$ also preserves $T_{\text {odd }}\left(\right.$ resp. $\left.T_{\text {even }}\right)$.

Accordingly, define finite subsets $\Phi_{\text {even }}^{\prime}, \Phi_{\text {even }}, \Phi_{\text {odd }}^{\prime}, \Phi_{\text {odd }} \subset G$ as follows:

(1) $\Phi_{\text {even }}^{\prime}$ consists of products $h_{j}^{-1} g_{i}, g_{i} \in G_{x_{i}}, h_{j} \in G_{x_{j}}$ with either (a) $i \neq j$ and $g_{i} \in G_{e_{\text {even }(i)}}$ and $h_{j} \in G_{e_{\text {even }(j)}}$, or (b) $i=j$ and $h_{i}^{-1} g_{i} \in G_{e_{e v e n(i)}}$.

(2) $\Phi_{\text {even }}:=\left\{h_{j}^{-1} g_{i}: g_{i} \in G_{x_{i}}, h_{j} \in G_{x_{j}}\right\} \backslash \Phi_{\text {even }}^{\prime}$.

(3) $\Phi_{\text {odd }}^{\prime}$ consists of products $h_{j}^{-1} g_{i}, g_{i} \in G_{x_{i}}, h_{j} \in G_{x_{j}}$ with either (a) $i \neq j$ and $g_{i} \in G_{e_{\text {odd }(i)}}$ and $h_{j} \in G_{e_{\text {odd }(j)}}$, or (b) $i=j$ and $h_{i}^{-1} g_{i} \in G_{e_{o d d(i)}}$.

(4) $\Phi_{\text {odd }}:=\left\{h_{j}^{-1} g_{i}: g_{i} \in G_{x_{i}}, h_{j} \in G_{x_{j}}\right\} \backslash \Phi_{\text {odd }}^{\prime}$.

Observe that $\Phi_{\text {even }}^{\prime}, \Phi_{\text {odd }}^{\prime}$ are contained in the subgroups $G_{\text {even }}, G_{\text {odd }}$ respectively.

Proposition $2.8 \Phi_{\text {odd }} \cap G_{\text {odd }}=\emptyset, \Phi_{\text {even }} \cap G_{\text {even }}=\emptyset$. In other words, among the products of the generators, only the "obvious" ones preserve the trees $T_{\text {odd }}$ and $T_{\text {even }}$. 
Proof We prove that $\Phi_{\text {odd }} \cap G_{o d d}=\emptyset$, the second assertion is proved by relabeling. We have to show that

$$
h_{j}^{-1} g_{i} T_{\text {odd }}=T_{\text {odd }} \Rightarrow h_{j}^{-1} g_{i} \in \Phi_{\text {odd }}^{\prime} .
$$

Throughout the proof we use the metric $\tau$ on $X$. We begin with the following

Observation 2.9 Let $g_{l} \in G_{x_{l}} \backslash G_{e_{\text {odd }(l)}}$ and $z$ be a vertex of $g_{l}(F)$. Then there is a geodesic segment $\sigma=\overline{z z^{\prime}} \subset(X, \tau)$ from $z$ to a point $z^{\prime} \in \Gamma_{\text {odd }}$, which intersects $T_{\text {odd }}$ orthogonally (at the point $z^{\prime}$ ) and which is entirely contained in $F \cup g_{l}(F)$. For instance, if $g_{l}(F) \cap F=\left\{x_{l}\right\}$, then the segment $\sigma$ equals $\overline{z x_{l}} \cup \overline{x_{l} m_{\text {odd }(l)}}$.

Moreover, unless $z \in g_{l}(F) \cap F$, the length of the segment $\sigma$ is strictly greater than 1. In particular, $d\left(z, T_{\text {odd }}\right)>1$.

Let $f:=h_{j}^{-1} g_{i}$ and assume that $f\left(T_{\text {odd }}\right)=T_{\text {odd }}$. Hence the edge $f\left(e_{\text {odd }(i)}\right) \subset X$ intersects $T_{\text {odd }}$ orthogonally in its midpoint $f\left(m_{\text {odd }(i)}\right)$. The segment $f\left(e_{\text {odd }(i)}\right)$ equals $\overline{z w}$ where $z:=h_{j}^{-1}\left(x_{i}\right)$ and $w:=f\left(x_{i+1}\right)$ (if $i$ is odd), and $w:=f\left(x_{i-1}\right)$ (if $i$ is even). In any case, $d\left(z, T_{\text {odd }}\right)=1$.

Suppose that $h_{j} \notin G_{\text {odd }(j)}$. Then, unless $z=x_{j}$, by applying Observation 2.9 to $l=j$, we obtain a contradiction with $d\left(z, T_{o d d}\right)>1$. Therefore $z=x_{j}$ and $i=j, f \in G_{x_{i}}$. Thus we apply Observation 2.9 to $l=j, g_{l}=f$ and the vertex $w \in f(F)$, and conclude that $w \in F$ as well. Then $f\left(e_{\text {odd }(i)}\right)=e_{\text {odd }(i)}$ and hence $f=h_{j}^{-1} g_{i} \in \Phi_{\text {odd }}^{\prime}$.

Hence we conclude that $h_{j} \in G_{o d d(j)}$. We now use the fact that $g_{i}^{-1} h_{j}\left(T_{o d d}\right)=$ $T_{\text {odd }}$ and reverse the roles of $g_{i}$ and $h_{j}$. The same argument as above then implies that either

(a) $g_{i} \in G_{\text {odd }(i)}$, or

(b) $i=j, f^{-1} \in G_{\text {odd }(j)}$.

In Case (a), $h_{j} \in G_{\text {odd }(j)}, g_{i} \in G_{o d d(i)}$ and thus $f \in \Phi_{\text {odd }}^{\prime}$; in Case (b) $i=j, f \in$ $G_{o d d(j)}$ and thus $f \in \Phi_{\text {odd }}^{\prime}$.

Corollary 2.10 For each $g \in \Phi_{\text {even }}, h \in \Phi_{\text {odd }}$ we have $g T_{\text {even }} \cap T_{\text {even }}=\emptyset$, $h T_{\text {odd }} \cap T_{\text {odd }}=\emptyset$.

Recall the following definition: 
Definition 2.11 A group $G$ is said to satisfy LERF property with respect to a subgroup $H \subset G$ if one of the following equivalent conditions holds:

(a) For each finite subset $F \subset G \backslash H$ there exists a homomorphism $\phi: G \rightarrow \bar{G}$, where $\bar{G}$ is a finite group and $\phi(H) \cap \phi(F)=\emptyset$.

(b) For each finite subset $F \subset G \backslash H$ there exists a finite index subgroup $G^{\prime} \subset G$ so that $H \subset G^{\prime}$ and $F \cap G^{\prime}=\emptyset$.

Note that for $H=\{1\}$ the above definition amounts to residual finiteness of $G$.

Corollary 2.12 There is an epimorphism $\phi: G \rightarrow \bar{G}$ where $\bar{G}$ is a finite group and $\phi\left(\Phi_{\text {even }}\right) \cap \phi\left(G_{\text {even }}\right)=\emptyset, \phi\left(\Phi_{\text {odd }}\right) \cap \phi\left(G_{\text {odd }}\right)=\emptyset$.

Proof According to 24, the group $G$ satisfies the LERF property with respect to each quasi-convex subgroup. Thus there are finite quotients

$$
\phi^{\prime}: G \rightarrow \bar{G}^{\prime}, \phi^{\prime \prime}: G \rightarrow \bar{G}^{\prime \prime}
$$

so that $\phi^{\prime}\left(G_{\text {even }}\right) \cap \phi^{\prime}\left(\Phi_{\text {even }}\right)=\emptyset, \phi^{\prime}\left(G_{\text {odd }}\right) \cap \phi^{\prime}\left(\Phi_{\text {odd }}\right)=\emptyset$. Then define the homomorphism $\phi=\left(\phi^{\prime}, \phi^{\prime \prime}\right): G \rightarrow \bar{G}^{\prime} \times \bar{G}^{\prime \prime}$ and let the group $\bar{G}$ be the image of $\phi$.

Let $q: X \rightarrow \bar{X}:=X / \operatorname{Ker}(\phi)$ denote the quotient map; the group $G$ acts on the compact complex $\bar{X}$ through the quotient group $\bar{G}$. We let $\bar{T}_{\text {even }}, \bar{T}_{\text {odd }}$ denote the projections of the trees $T_{\text {even }}, T_{\text {odd }}$ to the complex $\bar{X}$.

Lemma 2.13 Suppose that $g_{i} \in G_{x_{i}}, h_{j} \in G_{x_{j}}$ and $g_{i} \bar{T}_{\text {even }} \cap h_{j} \bar{T}_{\text {even }} \neq \emptyset$ (resp. $g_{i} \bar{T}_{\text {odd }} \cap h_{j} \bar{T}_{\text {odd }} \neq \emptyset$ ). Then $h_{j}^{-1} g_{i} \in \Phi_{\text {even }}^{\prime}$ (resp. $h_{j}^{-1} g_{i} \in \Phi_{\text {odd }}^{\prime}$ ).

Proof If $g_{i} \bar{T}_{\text {even }} \cap h_{j} \bar{T}_{\text {even }} \neq \emptyset$ then $g_{i} \bar{T}_{\text {even }}=h_{j} \bar{T}_{\text {even }}$. It follows that $k h_{j}^{-1} g_{i} \in$ $G_{\text {even }}$ for some $k \in \operatorname{Ker}(\phi)$; thus $\phi\left(h_{j}^{-1} g_{i}\right) \in \phi\left(G_{\text {even }}\right)$ which implies that $h_{j}^{-1} g_{i} \in \Phi_{\text {even }}^{\prime}$. The argument for $\bar{T}_{\text {odd }}$ is the same.

The graphs $\bar{T}_{\text {even }}, \bar{T}_{\text {odd }}$ determine finite subsets $S_{\text {even }}, S_{\text {odd }}$ of the set $\operatorname{Edges}(\bar{X})$ consisting of those edges in $\bar{X}$ which intersect $\bar{T}_{\text {even }}, \bar{T}_{\text {odd }}$ nontrivially. Let $\xi, \eta$ denote the characteristic functions of the subsets $S_{\text {even }}, S_{\text {odd }} \subset \operatorname{Edges}(\bar{X})$, normalized to have unit norm in the (finite-dimensional) Hilbert space $H:=$ $L^{2}(\operatorname{Edges}(\bar{X}))$. The group $G$ acts on $H$ by precomposition. We let $V \subset H$ denote the span of the subset $G \cdot\{\xi, \eta\} \subset H$ and let $p$ be the dimension of $V$. 
Corollary 2.14 (1) The subgroups $G_{\text {even }}, G_{\text {odd }}$ fix the vectors $\xi, \eta \in V$ respectively.

(2) The set

$$
\Sigma=\left\{g^{*}(\xi), g^{*}(\eta): g \in G_{x_{1}} \cup \ldots G_{x_{n}}\right\}
$$

is an orthonormal system in $V$.

(3) For all $g, h \in G_{x_{1}} \cup \ldots G_{x_{n}}$ :

(a) $g^{*}(\xi) \neq h^{*}(\eta)$.

(b) $g^{*}(\xi)=h^{*}(\xi)\left(\right.$ resp. $\left.g^{*}(\eta)=h^{*}(\eta)\right)$ iff $h^{-1} g \in \Phi_{\text {even }}^{\prime}\left(\right.$ resp. $\left.h^{-1} g \in \Phi_{\text {odd }}^{\prime}\right)$.

Let $L k_{x_{i}}^{\circ}$ denote the vertex set of the link of $x_{i}$ in $X$.

Corollary 2.15 The representation $G \curvearrowright V$ contains subrepresentations

$$
G_{x_{i}} \curvearrowright \operatorname{Vect}\left(L k_{x_{i}}^{\circ}\right)
$$

so that the orthonormal vectors $G_{x_{i}} \cdot\{\xi, \eta\}$ are identified with the vectors $G_{x_{i}} \cdot L k_{x_{i}}^{\circ}$

Proof Let us consider the case when $i$ is odd, since the other case is analogous. Observe that the stabilizer in $G_{x_{i}}$ of the vector $\xi \in V$ (resp. $\eta \in V$ ) is the group $G_{x_{i}} \cap G_{\text {even }}=G_{e_{\text {even }(i)}}$ (resp. $G_{x_{i}} \cap G_{\text {odd }}=G_{e_{\text {odd }(i)}}$ ); the stabilizer in $G_{x_{i}}$ of the vector $f_{i}^{-}$is $G_{e_{\text {even }(i)}}$. Thus we construct an isometric embedding $V \operatorname{ect}\left(L k_{x_{i}}^{\circ}\right) \rightarrow V$ by sending $f_{i}^{-}, f_{i}^{+}$to $\xi, \eta$ respectively, and then extending this map equivariantly to the orthonormal basis $L k_{x_{i}}^{\circ}$.

\section{$3 \quad$ Hyperbolic trigonometry}

Consider a regular right-angled hyperbolic $n$-gon $F \subset \mathbb{H}^{2}(n \geq 5)$. Let $a_{n}$ denote its side-length, $\rho_{n}$ the radius of the circumscribed circle, $r_{n}$ the radius of the inscribed circle, $b_{n}$ the length of the shortest diagonal in $F$ (ie, a diagonal which cuts out a triangle from $F$ ); see Figure 1. We then have:

$$
\begin{gathered}
\cosh \left(a_{n}\right)=1+2 \cos \left(\frac{2 \pi}{n}\right), \quad \cosh \left(\frac{a_{n}}{2}\right)=\sqrt{2} \cos \left(\frac{\pi}{n}\right), \quad \cosh \left(b_{n}\right)=\cosh ^{2}\left(a_{n}\right), \\
\cosh \left(r_{n}\right)=\frac{1}{\sqrt{2} \sin \left(\frac{\pi}{n}\right)}, \quad \cosh \left(\rho_{n}\right)=\cosh \left(r_{n}\right) \cosh \left(\frac{a_{n}}{2}\right) .
\end{gathered}
$$

Note that $a_{n}, b_{n}, r_{n}, \rho_{n}$ are strictly increasing functions of $n$. 


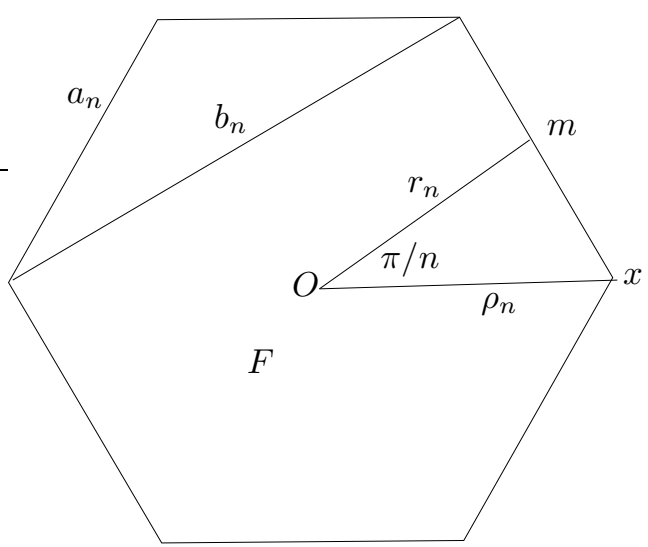

Figure 1: Geometry of a hyperbolic $n$-gon

Consider a Lambert quadrilateral $Q$ with one zero angle: $Q$ is a quadrilateral in $\mathbb{H}^{2}$ with one ideal vertex (where $Q$ has zero angle) and three finite vertices where the angles are $\pi / 2$ (Figure 2). Let $x, y$ denote the lengths of the finite sides of $Q$. Then

$$
\sinh (x) \sinh (y)=\cos (0)=1
$$

(see [2, 7.17.1]), or, equivalently

$$
\cosh ^{2}(x) \cosh ^{2}(y)=\cosh ^{2}(x)+\cosh ^{2}(y) .
$$

Thus, if we have two segments $E=\overline{x x^{\prime}}, E^{\prime}=\overline{x^{\prime} x^{\prime \prime}}$ in $\mathbb{H}^{2}$ which intersect at the point $x^{\prime}$ where they meet orthogonally, then the necessary and sufficient condition for $\operatorname{Bis}(E) \cap \operatorname{Bis}\left(E^{\prime}\right)=\emptyset$ is

$$
\cosh ^{2}(|E| / 2) \cosh ^{2}\left(\left|E^{\prime}\right| / 2\right) \geq \cosh ^{2}(|E| / 2)+\cosh ^{2}\left(\left|E^{\prime}\right| / 2\right),
$$

equivalently

$$
\sinh (|E| / 2) \sinh \left(\left|E^{\prime}\right| / 2\right) \geq 1 .
$$

We will refer to these inequalities as the disjoint bisectors test.

Lemma 3.1 Suppose that $E, E^{\prime} \subset \mathbb{H}^{2}$ are segments which meet orthogonally at a vertex, where $|E|=2 \rho_{n}$ and $\left|E^{\prime}\right|=a_{n}$. Then

$$
\emptyset=\overline{\operatorname{Bis}(E)} \cap \overline{\operatorname{Bis}\left(E^{\prime}\right)} \subset \overline{\mathbb{H}}^{2},
$$

provided that $n \geq 7$; in case $n=6$ we have:

$$
\overline{\operatorname{Bis}(E)} \cap \overline{\operatorname{Bis}\left(E^{\prime}\right)} \subset \partial_{\infty} \mathbb{H}^{2},
$$

is a point at infinity. 


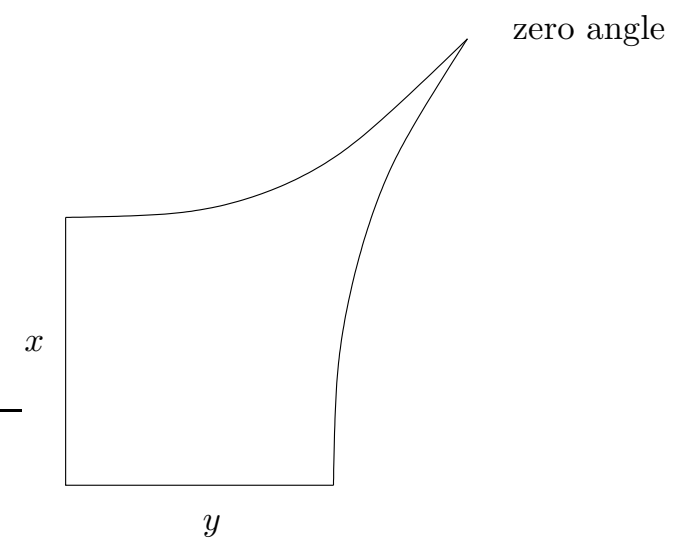

Figure 2: A Lambert quadrilateral

Proof Applying the disjoint bisectors test to $|E|=2 \rho_{6},\left|E^{\prime}\right|=a_{6}$ we get the equality. Hence the bisectors meet at infinity in case $n=6$ and are within positive distance from each other if $n \geq 7$.

Lemma 3.2 Suppose that $E, E^{\prime} \subset \mathbb{H}^{2}$ are segments which meet orthogonally at a vertex, where $|E|=b_{n}$ and $\left|E^{\prime}\right|=a_{n}, n \geq 7$. Then

$$
\emptyset=\overline{\operatorname{Bis}(E)} \cap \overline{\operatorname{Bis}\left(E^{\prime}\right)} \subset \overline{\mathbb{H}}^{2} \text {. }
$$

Proof Since $b_{n} \geq b_{7}, a_{n} \geq a_{7}$ it suffices to prove lemma in case $n=7$. Note that $a_{7}>a_{6}$ and

$$
b_{7} \approx 2.302366350>2 \rho_{6}=2.292431670
$$

Hence the assertion follows from Lemma 3.1

Below is another application of the disjoint bisectors test. Consider three segments $s, s^{\prime}, s^{\prime \prime}$ in $\mathbb{H}^{3}$ of the length $x, y, x$ respectively, which are mutually orthogonal and so that $s \cap s^{\prime}=p, s^{\prime} \cap s^{\prime \prime}=q, s^{\prime}=\overline{p q}$, see Figure 3 .

Corollary 3.3 If $x=b_{n}, y=a_{n}, n \geq 5$, then $\overline{\operatorname{Bis}(s)} \cap \overline{\operatorname{Bis}\left(s^{\prime \prime}\right)}=\emptyset$.

Proof It suffices to prove the corollary for $n=5$. We first compute the length $z=2 t$ of the segment $s^{\prime \prime \prime}$ coplanar to $s$ and $s^{\prime}$ such that $\operatorname{Bis}(s)=\operatorname{Bis}\left(s^{\prime \prime \prime}\right)$. By considering the Lambert's quadrilateral with angle $\phi$ we get:

$$
\left\{\begin{array}{c}
\cosh (t) \sin (\phi)=\cosh (x / 2) \\
\cosh (y) \sin (\phi)=\cosh (h) \\
\sinh (x / 2) \sinh (h)=\cos (\phi)
\end{array}\right.
$$




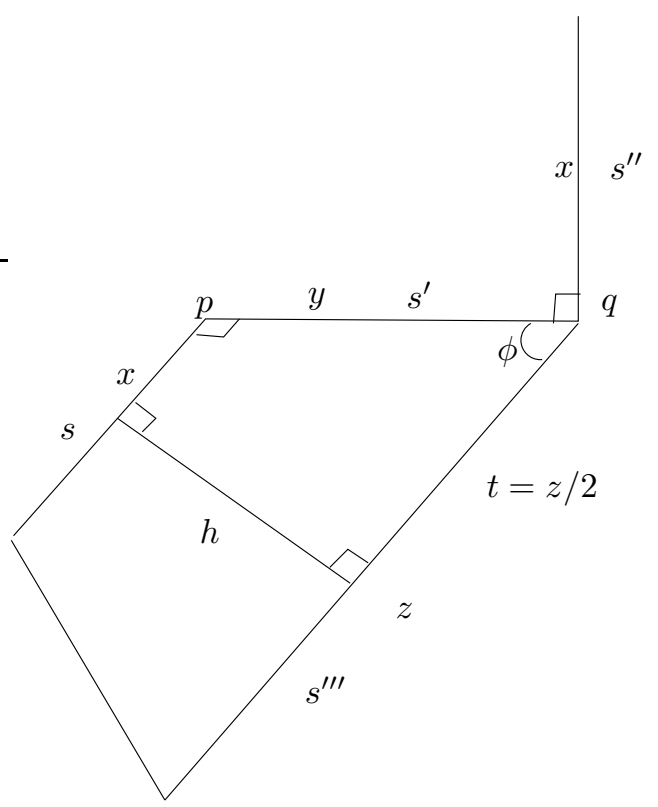

Figure 3: Three orthogonal segments

Thus

$$
\cosh (t)=\frac{\cosh (y) \cosh (x / 2)}{\cosh (h)} .
$$

On the other hand, the last two equations in the above system imply that

$$
\cosh (h)=\frac{\cosh (x / 2)}{\sqrt{\sinh ^{2}(x / 2)+1 / \cosh ^{2}(y)}} .
$$

Therefore

$$
\cosh (z / 2)=\sqrt{1+\sinh ^{2}(x / 2) \cosh ^{2}(y)} .
$$

By applying the disjoint bisectors test to $s^{\prime \prime \prime}, s^{\prime \prime}$ we get:

$$
\operatorname{Bis}(s) \cap \operatorname{Bis}\left(s^{\prime \prime}\right)=\emptyset \Longleftrightarrow \sinh ^{2}(x / 2) \cosh (y) \geq 1 .
$$

Lastly, we have:

$$
\cosh \left(a_{5}\right) \approx 1.6180, \quad \sinh \left(b_{5} / 2\right) \approx 1.85123 .
$$

Therefore $\sinh ^{2}\left(b_{5} / 2\right) \cosh \left(a_{5}\right)>1$. 


\section{Quasi-isometric maps of polygonal complexes}

Suppose that $X$ is a simply-connected 2-dimensional regular cell complex which is equipped with a path-metric so that:

1. Each face is isometric to a right-angled regular $n$-gon in $\mathbb{H}^{2}$ (of course, $n \geq 5)$.

2. The complex $X$ is negatively curved, ie, for each vertex $x \in X$ the length of the shortest embedded loop in $L k_{x}(X)$ is at least $2 \pi$.

Theorem 4.1 Suppose that $\mu: X \rightarrow \mathbb{H}^{p}$ is a continuous map which is a (totally-geodesic) isometric embedding on each face of $X$. We also assume that for each pair of faces $F^{\prime}, F^{\prime \prime} \subset X$ which intersect nontrivially a common face $F \subset X$, we have:

$$
\operatorname{Span}\left(\mu\left(F^{\prime}\right)\right) \perp \operatorname{Span}\left(\mu\left(F^{\prime \prime}\right)\right) .
$$

Then $\mu$ is a quasi-isometric embedding.

Proof Throughout the proof we will be using the notation $a_{n}, b_{n}, \rho_{n}$ for various distances in a regular right-angled hyperbolic $n$-gon, see Section 3 ,

Since the inclusion $X^{(1)} \hookrightarrow X$ is a quasi-isometry, it suffices to check that $X^{(1)} \stackrel{\mu}{\rightarrow} \mathbb{H}^{p}$ is a quasi-isometric embedding. Since $\mu$ is $1-$ Lipschitz, it is enough to show that $d(\mu(z), \mu(w)) \geq C \cdot d(z, w)$ for some $C=C(X)>0$ and all $z, w \in X^{(0)}$. We first give a proof in case $n \geq 6$ and then explain how to modify it for $n=5$.

Let $\widetilde{\gamma} \subset X^{(1)}$ be an (oriented) geodesic segment connecting $z$ to $w$. We start by replacing (in case when $n$ is even) each subsegment of $\widetilde{\gamma}$ connecting antipodal points in a face $F$ of $X$ with a geodesic segment within $F$. We will call the resulting (oriented) curve $\gamma \subset X$. Clearly,

$$
\operatorname{Length}(\mu(\gamma)) \leq \operatorname{Length}(\mu(\widetilde{\gamma})) \leq \frac{n}{2} \operatorname{Length}(\mu(\gamma)),
$$

so it suffices to get a lower bound on Length $(\mu(\gamma))$. We will refer to the edges of $\gamma$ connecting antipodal points of faces as diagonals in $\gamma$.

Remark 4.2 Suppose that $\overline{x x^{\prime}}, \overline{x^{\prime} x^{\prime \prime}}$ are (distinct) diagonals in $\gamma$, contained in faces $F, F^{\prime}$ respectively. Then $F \cap F^{\prime}=\left\{x^{\prime}\right\}$ : Otherwise $\widetilde{\gamma} \subset X^{(1)}$ would not be a geodesic as there exists a shorter path along the boundaries of $F$ and $F^{\prime}$; see Figure 4. In particular, $\mu\left(\overline{x x^{\prime}}\right) \perp \mu\left(\overline{x^{\prime} x^{\prime \prime}}\right)$ in $\mathbb{H}^{p}$. 


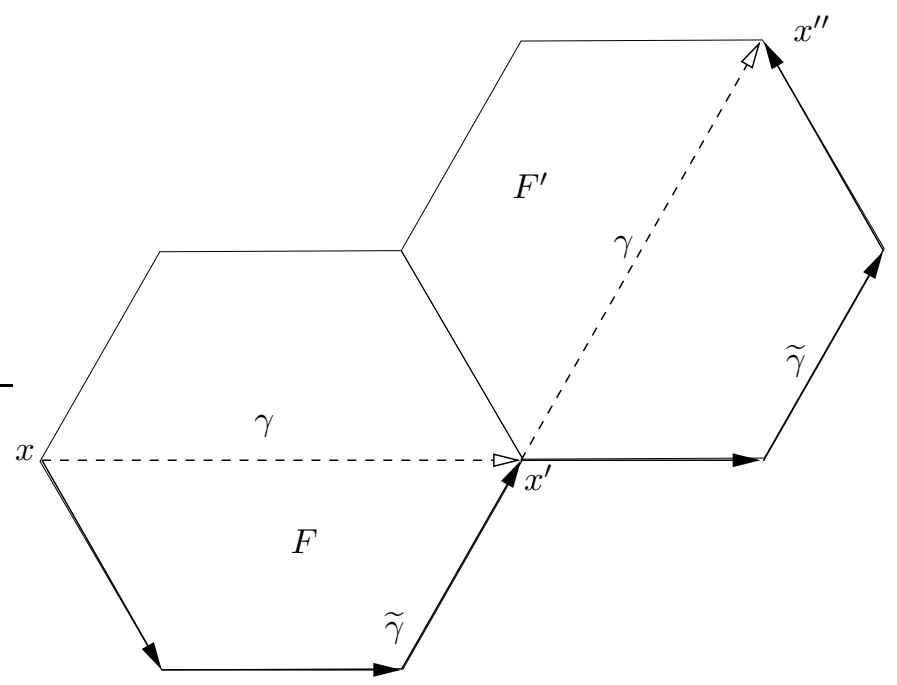

Figure 4: Diagonals

We will regard $\gamma$ as concatenation of consecutive segments $e_{0}, e_{1}, \ldots$ We define a collection $\mathcal{B E}(\gamma)$ of bisected edges $E_{i}$ in $\gamma$ inductively as follows:

(1) Let $E_{0}=e_{0} \subset \gamma$ be the first edge of $\gamma$.

(2) Suppose that $E_{i}=e_{j} \subset \gamma$ was chosen, $i \geq 0$. We will take as $E_{i+1}=e_{k}$, $k>i$, the first edge on $\gamma$ following $E_{i}$ which satisfies two properties:

(a) If $e_{i} \cap e_{k} \neq \emptyset$ then either $e_{i}$ or $e_{k}$ is a diagonal.

(b) $e_{i}, e_{k}$ do not belong to a common face in $X$.

Proposition 4.3 Suppose that $n \geq 6$. Then the edges $E_{i}, i=0,1, \ldots$ in $\gamma$ satisfy the following:

(1) There exists a constant $c=c(X)$ such that $d\left(E_{i}, E_{i+1}\right) \leq c$ in $X$.

(2) $\overline{\operatorname{Bis}\left(\mu\left(E_{i}\right)\right)} \cap \overline{\operatorname{Bis}\left(\mu\left(E_{i+1}\right)\right)} \subset \overline{\mathbb{H}}^{p}$ is empty unless $n=6$ and either $E_{i}$, or $E_{i+1}$ is not a diagonal. In case $n=6$ and at least one of these segments is not a diagonal, the bisectors $\operatorname{Bis}\left(\mu\left(E_{i}\right)\right), \operatorname{Bis}\left(\mu\left(E_{i+1}\right)\right) \subset \mathbb{H}^{p}$ are disjoint but have a common ideal point in $\partial_{\infty} \mathbb{H}^{p}$.

(3) For all edges $e_{j} \subset \gamma$ between $E_{i}, E_{i+1}$, their images $\mu\left(e_{j}\right)$ are disjoint from $\operatorname{Bis}\left(\mu\left(E_{i}\right)\right) \cup \operatorname{Bis}\left(\mu\left(E_{i+1}\right)\right)$. 


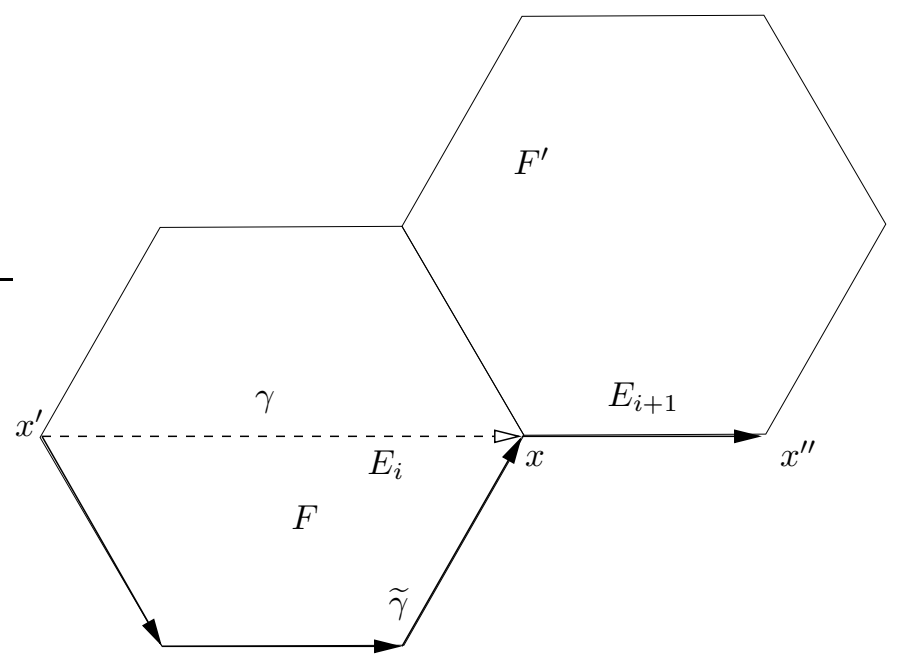

Figure 5: Diagonal and an edge

Proof (1) It is clear from the construction, that $E_{i}, E_{i+1}$ are separated by at most $n / 2$ edges on $\gamma$. Hence the first assertion follows.

(2) There are several cases we have to consider.

(a) Suppose that either $E_{i}$ or $E_{i+1}$ is a diagonal (see Figure 5) of the length $2 \rho_{n}$ in the notation of section 3. Then these segments share a common vertex $x^{\prime}$ and it follows that $\mu\left(E_{i}\right) \perp \mu\left(E_{i+1}\right)$ (see the Remark above). The worst case occurs when $n=6$ and one of the segments is an edge of a face of $X$ : The bisectors $\operatorname{Bis}\left(\mu\left(E_{i}\right)\right), \operatorname{Bis}\left(\mu\left(E_{i+1}\right)\right)$ are disjoint in $\mathbb{H}^{p}$ but have a common ideal point (see Lemma 3.1). Since, as $n$ increases, both side-lengths and lengths of diagonals in regular right-angled $n$-gons in $\mathbb{H}^{2}$ strictly increase, it follows that

$$
\overline{\operatorname{Bis}\left(\mu\left(E_{i}\right)\right)} \cap \overline{\operatorname{Bis}\left(\mu\left(E_{i+1}\right)\right)}=\emptyset, \forall n \geq 7 .
$$

(b) Consider now the case when neither $E_{i}=e_{k}$ nor $E_{i+1}$ is a diagonal, $E_{i}$ is contained in a face $F$ and there exists at least one edge (say, $e_{k+1}$ ) between $E_{i}, E_{i+1}$ which is contained in the face $F$. Then, by the construction, $E_{i+1}=\overline{x^{\prime} x^{\prime \prime}}$ is not contained in $F$ but shares the common point $x^{\prime}$ with $F$. Thus $\mu\left(E_{e+1}\right) \perp \mu(F)$.

Observe now that there is a vertex $y \in F$ such that the segments $\overline{y x^{\prime}}$ and $E_{i}$ have the same bisector in $F$. To find this vertex simply apply the reflection in $\operatorname{Bis}\left(E_{i}\right)$ to the vertex $x^{\prime}$ : This symmetry preserves $F$ and sends the vertex $x^{\prime}$ 


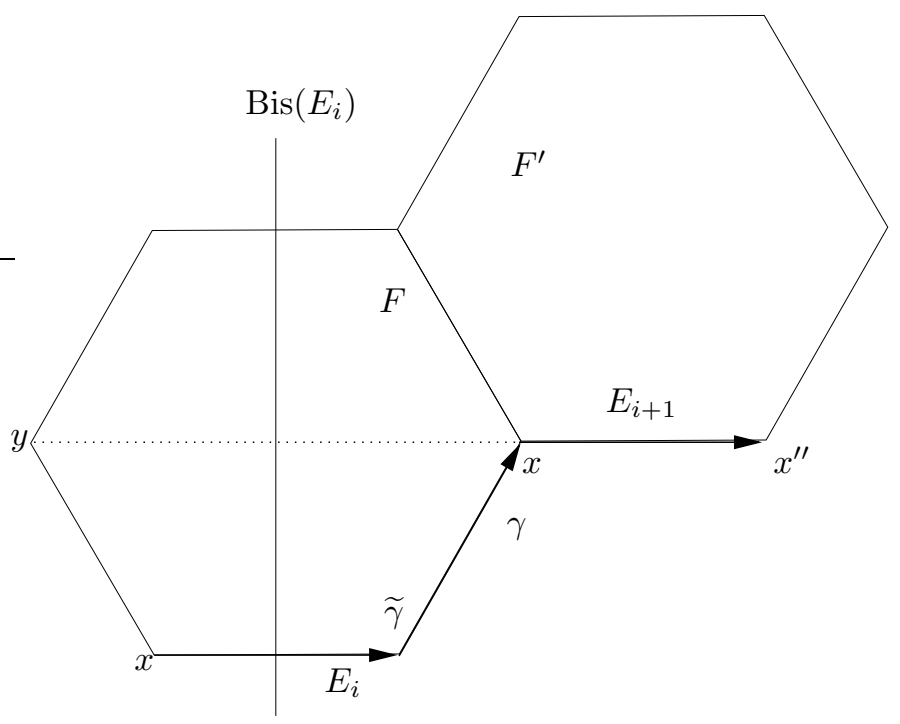

Figure 6: Bisectors

to a vertex $y \in F$. See Figure 6 . Since $x, x^{\prime} \in F$ are not antipodal, $\overline{y x^{\prime}}$ is not an edge of $F$.

Clearly, $\operatorname{Bis}\left(\mu\left(\overline{y x^{\prime}}\right)\right)=\operatorname{Bis}\left(\mu\left(E_{i}\right)\right)$. Hence the problem reduces to verifying that the bisectors $\operatorname{Bis}\left(\mu\left(\overline{y x^{\prime}}\right)\right)$, $\operatorname{Bis}\left(\mu\left(E_{i+1}\right)\right.$ ) (or their closures in $\overline{\mathbb{H}}^{p}$ ) are disjoint. We note that in case $n=6$ the segment $\overline{y x^{\prime}}$ connects antipodal points in $F$; hence the proof in this case reduces to (a).

Assume now that $n \geq 7$, then, since $\overline{y x^{\prime}}$ is not an edge of $F,\left|x y^{\prime}\right| \geq b_{n} \geq b_{7}$, $\left|x^{\prime} x^{\prime \prime}\right|=a_{n} \geq a_{7}$ and

$$
\overline{\operatorname{Bis}\left(\mu\left(E_{i}\right)\right)} \cap \overline{\operatorname{Bis}\left(\mu\left(E_{i+1}\right)\right)}=\emptyset,
$$

follows from Lemma 3.2

(c) The last case to consider is when $E_{i}, E_{i+1}$ are not diagonals and they are separated by exactly one edge $e \subset \gamma$ (this edge cannot be a diagonal in this case), which is not contained in a common face with $E_{i}$ nor with $E_{i+1}$. Then the edges

$$
\mu\left(E_{i}\right), \mu(e), \mu\left(E_{i+1}\right) \subset \mathbb{H}^{p}
$$

intersect orthogonally. The lengths of these edges are equal to $a(n) \geq a(6)$. Hence, (as in Case (b)) we replace $\mu\left(E_{i}\right)$ with a segment $s$ of the length $\geq 2 \rho_{6}$ which meets $\mu\left(E_{i+1}\right)$ orthogonally at the point $\mu(e) \cap \mu\left(E_{i+1}\right)$. Therefore, by 
applying again Lemma 3.1 the bisectors $\operatorname{Bis}\left(\mu\left(E_{i}\right)\right), \operatorname{Bis}\left(\mu\left(E_{i+1}\right)\right)$ are disjoint; their closures in $\overline{\mathbb{H}}^{p}$ are disjoint provided $n \geq 7$.

This proves the second assertion of the Proposition. The third assertion is clear from the construction: For instance, in Case (b) the edges $e_{j}$ between $E_{i}, E_{i+1}$ are all contained in the face $F$. Therefore they are disjoint from the bisector of $E_{i}$ within $F$, which implies the assertion about their images in $\mathbb{H}^{p}$. On the other hand, the edge $\mu E_{i+1}$ is orthogonal to $\mu(F)$, hence $\operatorname{Bis}\left(\mu E_{i+1}\right)$ is disjoint from $\mu F$.

Now, let us finish the proof that $\mu$ is a quasi-isometric embedding. Suppose that $\gamma \subset X$ has length $L$, then the subset $\mathcal{B E}(\gamma)$ consists of $\ell \approx L / c$ bisected edges $E_{i}$ (here $c$ is the constant from Proposition 4.3. Part 1). Hence the geodesic segment $\gamma^{*}=\overline{\mu(z) \mu(w)}$ in $\mathbb{H}^{p}$ connecting the end-points of $\mu(\gamma)$ crosses $\ell$ bisectors $\operatorname{Bis}\left(\mu E_{i}\right)$. In case $n \geq 7$, the bisectors $\operatorname{Bis}\left(\mu E_{i}\right), \operatorname{Bis}\left(\mu E_{i+1}\right)$ are separated by distance $\delta=\delta(X)>0$, hence the length of $\gamma^{*}$ is at least $\ell \delta$. Since $\ell \approx L / c$, we conclude that $d_{\mathbb{H}^{p}}(x, y) \geq$ Const $\cdot L / \delta$. It follows that $\mu$ is a quasi-isometry.

Now, consider the exceptional case $n=6$. We claim that for each $i$ the intersection points $\overline{\operatorname{Bis}\left(\mu E_{i}\right)} \cap \overline{\operatorname{Bis}\left(\mu E_{i+1}\right)}$ and $\overline{\operatorname{Bis}\left(\mu E_{i+2}\right)} \cap \overline{\operatorname{Bis}\left(\mu E_{i+1}\right)}$ are distinct. Given this, instead of the collection $\mathcal{B E}(\gamma)$ we would consider the collection of edges $E_{i} \in \mathcal{B E}(\gamma)$ for even $i$, then $\overline{\operatorname{Bis}\left(\mu E_{i}\right)} \cap \overline{\operatorname{Bis}\left(\mu E_{i+2}\right)}=\emptyset$ for all even $i$ and we are done by the same argument as for $n \geq 7$.

Case I We begin with the case when $E_{i} \subset F_{i}, E_{i+2} \subset F_{i+2}$ are diagonals and $E_{i+1} \subset F_{i}$ is not. (Here $F_{i}$ are faces of $X$.) Then $E_{i} \cap E_{i+1}, E_{i+2} \cap E_{i+1}$ are the end-points of $E_{i+1}$. Therefore

$$
\begin{gathered}
\xi_{i}=\overline{\operatorname{Bis}\left(\mu E_{i}\right)} \cap \overline{\operatorname{Bis}\left(\mu E_{i+1}\right)} \in \partial_{\infty} \operatorname{Span}\left(\mu\left(E_{i}\right) \cup \mu\left(E_{i+1}\right)\right), \\
\xi_{i+1}=\overline{\operatorname{Bis}\left(\mu E_{i+1}\right)} \cap \overline{\operatorname{Bis}\left(\mu E_{i+2}\right)} \in \partial_{\infty} \operatorname{Span}\left(\mu\left(E_{i+1}\right) \cup \mu\left(E_{i+2}\right)\right) .
\end{gathered}
$$

However, by the assumptions on $\mu$,

$$
\operatorname{Span}\left(\mu\left(F_{i+2}\right)\right) \perp \operatorname{Span}\left(\mu\left(F_{i}\right)\right)
$$

Thus

$$
\operatorname{Span}\left(\mu\left(E_{i}\right) \cup \mu\left(E_{i+1}\right)\right) \cap \operatorname{Span}\left(\mu\left(E_{i+1}\right) \cup \mu\left(E_{i+2}\right)\right)=\operatorname{Span}\left(\mu\left(E_{i+1}\right)\right) .
$$

Since it is clear that $\xi_{i} \notin \partial_{\infty} \operatorname{Span}\left(\mu\left(E_{i+1}\right)\right)$, we conclude that $\xi_{i} \neq \xi_{i+1}$ and the assertion follows. 
We will reduce the case of a general triple of edges $E_{i}, E_{i+1}, E_{i+2}$ to the Case I discussed above. We consider only one other case, the arguments in the rest of cases are identical to:

Case II Suppose that pairs of edges $E_{i} \subset F_{i}, E_{i+1} \subset F_{i+1}, E_{i+2} \subset F_{i+2}$ are as in Figure 7. We take the diagonals $D_{i} \subset F_{i}, D_{i+2} \subset F_{i+2}$ so that

$$
\operatorname{Bis}\left(\mu D_{i}\right)=\operatorname{Bis}\left(\mu E_{i}\right), \operatorname{Bis}\left(\mu D_{i+2}\right)=\operatorname{Bis}\left(\mu E_{i+2}\right) .
$$

Now the proof reduces to the Case I.

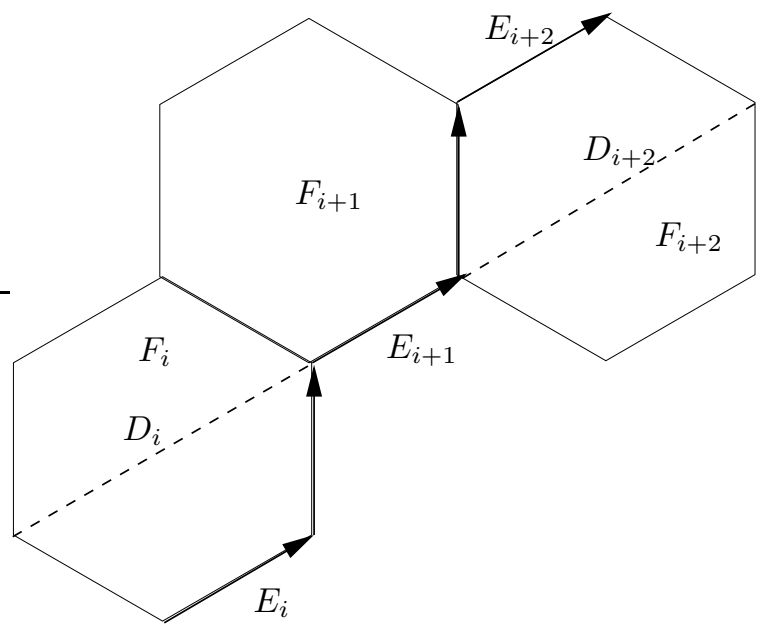

Figure 7

Finally, consider the case of pentagons (ie $n=5$ ). We define the collection $\mathcal{B E}(\gamma)$ of bisected edges $E_{i}$ as before. Let $E_{i}, E_{2}, E_{3}$ be consecutive bisected edges. We will see that $\operatorname{Bis}\left(\mu E_{1}\right) \cap \operatorname{Bis}\left(\mu E_{3}\right)=\emptyset$. Since $n=5$ we necessarily have: $E_{2}$ is separated by a unique edge $e \subset \gamma$ from $E_{1}$ and by a unique edge $e^{\prime} \subset \gamma$ from $E_{3}$, see Figure 8

Note that it could happen that there is no face $F_{1}$ which contains $E_{1}, e$, nor a face $F_{3}$ which contains $E_{3}, e^{\prime}$. However, in $\mathbb{H}^{p}$ there exists a unique regular right angled pentagon which contains the edges $\mu\left(E_{1}\right), \mu(e)\left(\operatorname{resp} . \mu\left(E_{3}\right), \mu\left(e^{\prime}\right)\right)$ in its boundary. Hence we will give a proof pretending that the corresponding face already exits in $X$. Observe that, similarly to our discussion above, the diagonals $D_{1} \subset F_{1}, D_{3} \subset F_{3}$ have the property that $\operatorname{Bis}\left(\mu D_{i}\right)=\operatorname{Bis}\left(\mu E_{i}\right)$, $i=1,3$. Thus it suffices to consider the triple of pairwise orthogonal segments: $\mu\left(D_{1}\right), \mu\left(E_{2}\right)$ and $\mu D_{3}$ in $\mathbb{H}^{p}$. The length of $\mu\left(E_{2}\right)$ equals $a_{5}$, the lengths of 


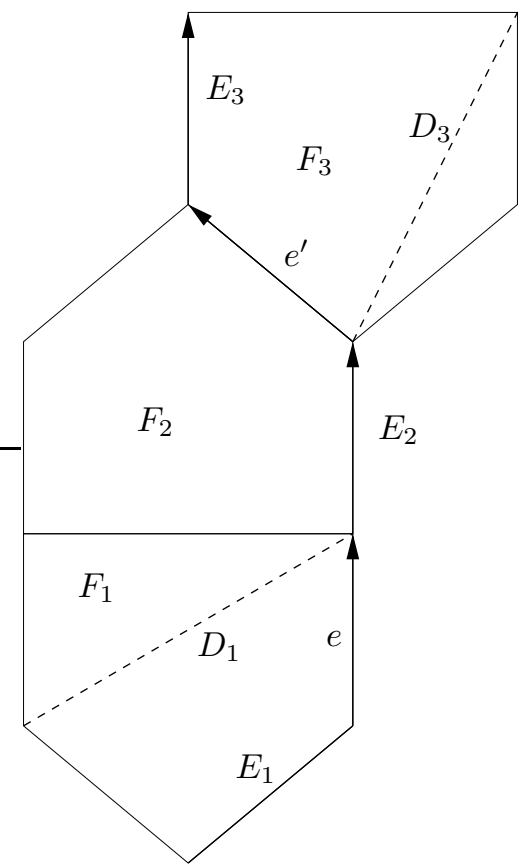

Figure 8

$D_{1}, D_{3}$ are equal to $b_{5}$, hence

$$
\overline{\operatorname{Bis}\left(\mu D_{1}\right)} \cap \overline{\operatorname{Bis}\left(\mu D_{3}\right)}=\emptyset
$$

by Corollary 3.3

\section{Proof of the main theorem}

In this section establish

Theorem 5.1 Suppose that $n=2 k$ is even. Then the group $G$ admits an isometric properly discontinuous convex-cocompact action $\rho: G \curvearrowright \mathbb{H}^{p}$, where $p<\infty$ depends on the polygon $\mathcal{P}$.

Proof Let $X$ be the universal cover of the polygon of groups $\mathcal{P}$. We first construct a representation $\rho: G \rightarrow \operatorname{Isom}\left(\mathbb{H}^{p}\right)$ for a certain $p$. We then produce a quasi-isometric $\rho$-equivariant embedding $\mu: X \rightarrow \mathbb{H}^{p}$. From this, via Lemma 
2.2 it will follow that $\rho: G \curvearrowright \mathbb{H}^{p}$ is an isometric properly discontinuous convexcocompact action.

Let $L k_{x}$ denote the link (in $X$ ) of the vertex $x$, similarly, let $L k_{e}$ denote the link of the edge $e$. Recall that $L k^{\circ}$ denotes the vertex set of a link $L k$. The set $L k_{x_{i}}^{\circ}$ contains two distinguished elements: $f_{i}^{+}, f_{i}^{-}$which correspond to the directions from $x_{i}$ toward $x_{i+1}$ and toward $x_{i-1}$ respectively. We define the subsets $L k_{x_{i}}^{\bullet}:=L k_{x_{i}}^{\circ} \backslash\left\{f_{i}^{+}, f_{i}^{-}\right\}$.

Observe that the directions $\eta_{i} \in L k_{x_{i}}^{\circ}, \eta_{i+1} \in L k_{x_{i+1}}^{\circ}$ belong to the boundary of a common face in $X$ if and only if there exists $g \in G_{e_{i}}$ so that

$$
\eta_{i}=g\left(f_{i}^{ \pm}\right), \eta_{i+1}=g\left(f_{i+1}^{ \pm}\right) .
$$

\section{Step 1: Construction of $\rho$}

It is clear that to construct a representation $\rho: G \rightarrow \operatorname{Isom}\left(\mathbb{H}^{p}\right)$ (for some $p$ ) we have to produce a collection of faithful representations

$$
\rho_{i}: G_{x_{i}} \rightarrow \operatorname{Isom}\left(\mathbb{H}^{p}\right)
$$

so that the following diagram commutes:

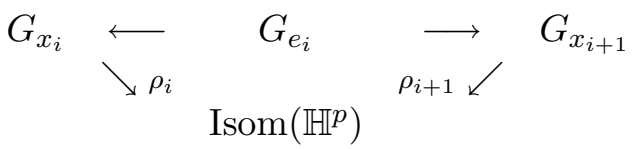

Embed $F$ isometrically (as a convex, regular, right-angled polygon) in the hyperbolic plane $\mathbb{H}^{2}$. Via this embedding we will identify the directions $f_{i}^{ \pm} \in L k_{x_{i}}$ with the unit vectors $\overrightarrow{f_{i}^{ \pm}} \in T_{x_{i}} \mathbb{H}^{2}$ which are tangent to the sides of $F$.

In what follows we will adopt the following convention. Given a number $p$ and a totally-geodesic embedding $\mathbb{H}^{2} \subset \mathbb{H}^{p}$, we observe that the normal bundle $N\left(\mathbb{H}^{2}\right)$ of $\mathbb{H}^{2}$ in $\mathbb{H}^{p}$ admits a canonical flat orthogonal connection (invariant under the stabilizer of $\mathbb{H}^{2}$ in $\left.\operatorname{Isom}\left(\mathbb{H}^{p}\right)\right)$. Thus, given normal vectors $\nu \in$ $N_{x}\left(\mathbb{H}^{2}\right), \nu^{\prime} \in N_{x^{\prime}}\left(\mathbb{H}^{2}\right)$, we have well-defined scalar product $\nu \cdot \nu^{\prime}$ and hence the notion of orthogonality $\nu \perp \nu^{\prime}$. Set $V_{i}:=T_{x_{i}} \mathbb{H}^{p}$ and let $N_{i} \subset V_{i}$ denote the orthogonal complement to $T_{x_{i}} \mathbb{H}^{2}$. We define $R_{i} \in \operatorname{Isom}\left(\mathbb{H}^{p}\right)$ to be the isometric reflection in the bisector of the edge $e_{i}$ of $F \subset \mathbb{H}^{p}$. Set $J_{i}:=R_{i-1} \circ \ldots \circ R_{1}$, for $i=2, \ldots, n+1$; observe that $J_{n+1}=I d$.

Remark 5.2 The fact that the identity $J_{n+1}=I d$ fails if $n$ is odd is one of the reasons why our construction requires $n$ to be an even number. An attempt to apply the constructions below to odd $n$ lead to a representation of a certain extension of the group $G$ rather than of $G$ itself. 
Proposition 5.3 Suppose that $n=2 k$ is even. Then there exists a natural number $p$ and a collection of faithful isometric linear actions $d \rho_{i}: G_{x_{i}} \curvearrowright V_{i}$, $i=1, \ldots, n$, so that the following hold:

(1) Each representation $d \rho_{i}$ contains a subrepresentaion $G_{x_{i}} \curvearrowright \operatorname{Vect}\left(\operatorname{Lk}_{x_{i}}^{\circ}\right)$, so that the unit basis vectors $f_{i}^{ \pm} \in L k_{x_{i}}^{\circ} \subset \operatorname{Vect}\left(L k_{x_{i}}^{\circ}\right)$ are identified with the vectors $\overrightarrow{f_{i}^{ \pm}} \in V_{i}$.

(2) Each reflection $R_{i}: \mathbb{H}^{p} \rightarrow \mathbb{H}^{p}$ induces an isomorphism of $\mathbb{R} G_{e_{i}}$-modules

$$
\left(V_{i}, d \rho_{i}\left(G_{e_{i}}\right)\right) \rightarrow\left(V_{i+1}, d \rho_{i+1}\left(G_{e_{i}}\right)\right) .
$$

(3) "Orthogonality": The spaces $\operatorname{Vect}\left(L k_{x_{i}}^{\bullet}\right) \subset N_{i}, \operatorname{Vect}\left(L k_{x_{j}}^{\bullet}\right) \subset N_{j}$ are mutually orthogonal, $|i-j| \geq 2, i, j \in\{1, \ldots, n\}$. If $j=i+1$ then we require orthogonality of the subspaces

$$
\operatorname{Vect}\left(L k_{x_{i}}^{\circ} \backslash G_{e_{i}} \cdot\left\{f_{i}^{+}, f_{i}^{-}\right\}\right) \subset N_{i}, \operatorname{Vect}\left(L k_{x_{i+1}}^{\circ} \backslash G_{e_{i}} \cdot\left\{f_{i+1}^{+}, f_{i+1}^{-}\right\}\right) \subset N_{i+1} .
$$

Remark 5.4 (1) The assumption that the number of sides of $F$ is even is used only in this part of the proof of the main theorem and very likely is just a technicality.

(2) The "orthogonality" property will be used to prove that the action $G \curvearrowright \mathbb{H}^{p}$ that we are about to construct, is discrete, faithful and convex-cocompact.

Before beginning the proof of the proposition we first make some observations (where we ignore the orthogonality issue). Suppose that we have constructed representations $d \rho_{i}$. We then "fold" these representations into a single orthogonal representation $G \curvearrowright V_{1}$ by composing each $d \rho_{i}$ with the composition of reflections $\left(R_{1}\right)_{*} \circ \ldots \circ\left(R_{i-1}\right)_{*}$, where $\left(R_{j}\right)_{*}$ is the isomorphism $O\left(V_{j+1}\right) \rightarrow O\left(V_{j}\right)$ which is induced by $\left.d R_{j}\right|_{x_{j+1}}$. Note that under the action $G \curvearrowright V_{1}$ the vectors $\vec{f}_{1}^{+}, \vec{f}_{1}^{-}$are fixed by the "odd" and "even" subgroups $G_{\text {odd }}, G_{\text {even }}$, respectively. Moreover, the representation $G \curvearrowright V_{1}$ contains subrepresentations $G_{x_{i}} \curvearrowright \operatorname{Vect}\left(L k_{x_{i}}^{\circ}\right)$.

Recall that in Corollary 2.14 we have constructed a finite-dimensional orthogonal representation $G \curvearrowright V$ which satisfies the same properties as above: It contains unit vectors $\xi, \eta$ fixed by $G_{\text {even }}, G_{\text {odd }}$ respectively, and it contains subrepresentations $G_{x_{i}} \curvearrowright \operatorname{Vect}\left(L k_{x_{i}}^{\circ}\right)$. Therefore, to construct the representations $d \rho_{i}$ we begin with the action $G \curvearrowright V$ (which we identify with an action $G \curvearrowright V_{1}$ ) and then "unfold" it (using compositions of reflections $R_{i}$ ) to a collection of representations $d \rho_{i}$. This is the idea of the proof of Proposition 5.3 
The reader familiar with bending deformations of representations of groups into $\operatorname{Isom}\left(\mathbb{H}^{p}\right)$ will notice that the "folding" and "unfolding" of representations discussed above is nothing but $\pi$-bending.

Proof We let $G \curvearrowright V$ denote the orthogonal representation constructed in Corollary 2.14, According to Corollary 2.15, the representation $G \curvearrowright V$ contains subrepresentations $G_{x_{i}} \curvearrowright V e c t\left(L k_{x_{i}}^{\circ}\right)$. Let $p$ denote the dimension of $V$.

Our goal is to construct isometries $\phi_{i}: V \rightarrow V_{i}:=T_{x_{i}} \mathbb{H}^{p}$. The actions $d \rho_{i}$ will be obtained by the conjugation:

$$
d \rho_{i}: G_{x_{i}} \curvearrowright V_{i}:=\phi_{i} \circ\left(G_{x_{i}} \curvearrowright V\right) \circ \phi_{i}^{-1} .
$$

First take an arbitrary isometry $\phi_{1}: V \rightarrow V_{1}$ sending the unit vectors $\xi, \eta \in V$ to the vectors $\vec{f}_{1}^{-}, \vec{f}_{1}^{+}$respectively. Now define isometries $\phi_{j}, j=2, \ldots, n+1$ by

$$
\phi_{i}=J_{i} \circ \phi_{1}
$$

ie

$$
\phi_{i+1}=R_{i} \circ \phi_{i}
$$

Note that $\phi_{n+1}=\phi_{1}$. Define the action $d \rho_{i}: G_{x_{i}} \curvearrowright V_{i}$ by conjugating via $\phi_{i}$ the action $G_{x_{i}} \curvearrowright V$.

The group $G_{e_{1}}$ fixes the vector $\eta \in V$, hence $G_{e_{1}}$ also fixes the vector $\phi_{1}(\eta)=$ $\vec{f}_{1}^{+}$. Thus

$$
\left.d R_{1} \circ d \rho_{1}\right|_{G_{e_{1}}}=\left.d \rho_{2}\right|_{G_{e_{1}}} .
$$

The same argument shows that

$$
\left.d R_{i} \circ d \rho_{i}\right|_{G_{e_{i}}}=\left.d \rho_{i+1}\right|_{G_{e_{i}}} .
$$

for all $i$. This proves (1) and (2). In what follows we will identify the spaces $\operatorname{Vect}\left(L k_{x_{i}}^{\circ}\right)$ with their images in $V_{i}, i=1, \ldots, n$.

We will check that the sets

$$
L k_{x_{i}}^{\circ} \backslash G_{e_{i}} \cdot\left\{f_{i}^{+}, f_{i}^{-}\right\} \subset N_{i}, L k_{x_{i+1}}^{\circ} \backslash G_{e_{i}} \cdot\left\{f_{i+1}^{+}, f_{i+1}^{-}\right\} \subset N_{i+1}
$$

are orthogonal to each other and will leave the remaining orthogonality assertion to the reader. Let

$$
v \in L k_{x_{i}}^{\circ} \backslash G_{e_{i}} \cdot\left\{f_{i}^{+}, f_{i}^{-}\right\} \subset N_{i}, w \in L k_{x_{i+1}}^{\circ} \backslash G_{e_{i}} \cdot\left\{f_{i+1}^{+}, f_{i+1}^{-}\right\} \subset N_{i+1} .
$$

In order to show that $v \perp w$ it suffices to verify that the corresponding vectors

$$
v, w \in \Sigma \subset V
$$


are distinct (recall that $\Sigma$ is an orthonormal system in $V$ ). If, say, $v \in G \cdot \xi, w \in$ $G \cdot \eta$ then $v \neq w$. Hence we will consider the case

$$
v=g^{*} \eta \in G_{x_{i}} \cdot \eta, w=h^{*} \eta \in G_{x_{i+1}} \cdot \eta .
$$

According to Corollary 2.14 if $g^{*}(\eta)=h^{*}(\eta)$ then $h^{-1} g \in \Phi_{\text {odd }}^{\prime}$. In our case, $g \in G_{x_{i}}, h \in G_{x_{i+1}}$. Then $h^{-1} g \in \Phi_{\text {odd }}^{\prime}$ means that either

(a) $g, h$ do not have a common fixed vertex of $F$ and $g \in G_{\text {odd }(i)}, h \in G_{\text {odd (i+1) }}$,

or

(b) $g, h$ fix the same vertex of $F$ and $g^{*}(\eta)=h^{*}(\eta)$.

In case (a), if $i$ is odd then $g, h \in G_{e_{i}}$, and hence $g^{*} \eta=g^{*} \eta$ corresponds to the vectors $f_{i}^{+} \in N_{i}, f_{i}^{-} \in N_{i+1}$. Therefore the equality $v=w$ implies that

$$
\phi_{i}(v) \in G_{e_{i}} \cdot \vec{f}_{i}^{+}, \phi_{i+1}(w) \in G_{e_{i}} \cdot \vec{f}_{i+1}^{-} .
$$

If $i$ is even then both $g \in G_{e_{i-1}}, h \in G_{e_{i+1}}$ fix the vector $\eta$. Therefore the equality $v=w$ implies that

$$
\phi_{i}(v)=\vec{f}_{i}^{-} \in G_{e_{i}} \cdot \vec{f}_{i}^{-}, \phi_{i+1}(w)=\vec{f}_{i+1}^{+} \in G_{e_{i}} \cdot \vec{f}_{i+1}^{+} .
$$

In case (b), we can assume that, say, $h \in G_{x_{i}} \cap G_{x_{i+1}}=G_{e_{i}}$. If $i$ is odd then $h^{*} \eta=\eta$ and therefore $g^{*} \eta=\eta$. Therefore the equality $v=w$ implies that

$$
\phi_{i}(v)=f_{i}^{+} \in G_{e_{i}} \cdot f_{i}^{+}, \quad \phi_{i+1}(w)=f_{i+1}^{-} \in G_{e_{i}} \cdot f_{i+1}^{-} .
$$

Lastly, assume that $i$ is even. Then the vector $v=g^{*}(\eta)=w=h^{*}(\eta)$ corresponds to the vectors

$$
\phi_{i}(w)=h\left(\vec{f}_{i}^{-}\right) \in G_{e_{i}} \cdot \vec{f}_{i}^{-} \subset N_{i}, \quad \phi_{i+1}(w)=h\left(\vec{f}_{i+1}^{+}\right) \in G_{e_{i}} \cdot \vec{f}_{i+1}^{+} \subset N_{i+1} .
$$

This proves the orthogonality assertion.

Now, once we have constructed linear orthogonal representations $d \rho_{i}: G_{x_{i}} \curvearrowright$ $V_{i}$, we extend them (by exponentiation) to isometric actions $\rho_{i}: G_{x_{i}} \curvearrowright \mathbb{H}^{p}$, which fix the points $x_{i}, i \in 1, \ldots, n$. Observe that for each $i$ the group $\rho_{i}\left(G_{e_{i}}\right)$, resp. $\rho_{i}\left(G_{e_{i-1}}\right)$, fixes the edge $e_{i}$, resp. $e_{i-1}$, of the polygon $F \subset \mathbb{H}^{2}$, since $d \rho_{i}\left(G_{e_{i}}\right), d \rho_{i}\left(G_{e_{i-1}}\right)$, fix the vectors $\overrightarrow{f_{i}^{+}}, \overrightarrow{f_{i}^{-}}$respectively. Hence the reflection $R_{i}$ commutes with the groups $\rho_{i}\left(G_{e_{i}}\right)$ and $\rho_{i+1}\left(G_{e_{i}}\right)$. Thus the representations $\rho_{i}, \rho_{i+1}: G_{e_{i}} \rightarrow \operatorname{Isom}\left(\mathbb{H}^{p}\right)$ are the same. Therefore the representations $\rho_{i}$ determine an isometric action $\rho: G \curvearrowright \mathbb{H}^{p}$. 


\section{Step 2: Discreteness of $\rho$}

We will construct a $\rho$-equivariant continuous mapping $\mu: X \rightarrow \mathbb{H}^{p}$ satisfying the assumptions of Theorem 4.1. Since such $\mu$ is necessarily a quasiisometric embedding (Theorem 4.1), by applying Lemma 2.2. we will conclude that $\rho: G \curvearrowright \mathbb{H}^{p}$ is properly discontinuous and convex-cocompact.

Recall that we have identified the face $F$ of $X$ with a regular right-angled hyperbolic polygon $F$ in $\mathbb{H}^{2}$, this defines the (identity) embedding $\mu: F \rightarrow \mathbb{H}^{2}$. Now, for each $g \in G$ we set

$$
\left.\mu\right|_{g F}:=\left.\rho(g) \circ \mu\right|_{F} .
$$

Let us check that this mapping is well-defined:

(1) If $g \in G_{F}$ then, by construction of $\rho, \rho(g)$ fixes the polygon $\mu(F)$ pointwise, hence $\left.\mu \circ g\right|_{F}=\left.\rho(g) \circ \mu\right|_{F}$ for $g \in G_{F}$.

(2) If $g \in G_{e}$, where $e$ is an edge of $\mathcal{P}$, then, by the construction, $\rho(g)$ fixes the edge $\mu(e)$ pointwise, hence $\left.\mu \circ g\right|_{e}=\left.\rho(g) \circ \mu\right|_{e}$ for $g \in G_{e}$.

(3) The same argument applies to $g \in G_{x}$, for the vertices $x \in F$.

Hence $\mu: X \rightarrow \mathbb{H}^{p}$ is well-defined and thus it is a $\rho$-equivariant, continuous mapping which is an isometric totally-geodesic embedding on each face of $X$.

Lastly, we check the orthogonality condition required by Theorem 4.1 By equivariance, it is clear that we only need to verify orthogonality for the faces $F^{\prime}, F^{\prime \prime} \subset X$ which are adjacent to the face $F \subset X$. We will see that this orthogonality condition will follow from the Assertion 3 of Proposition [5.3. There are several cases which may occur, we will check one of them and will leave the rest to the reader.

Suppose that $F^{\prime}=g_{i}(F), F^{\prime \prime}=g_{j}(F), g_{i} \in G_{x_{i}}, G_{j} \in G_{x_{j}}$ and

$$
F^{\prime} \cap F=\left\{x_{i}\right\}, \quad F^{\prime \prime} \cap F=\left\{x_{j}\right\},
$$

where $|i-j| \geq 2$. Then

$$
T_{x_{i}}\left(\mu F^{\prime}\right) \subset N_{i}, \quad T_{x_{j}}\left(\mu F^{\prime \prime}\right) \subset N_{j}
$$

and the vectors

$$
d g_{i}\left(\vec{f}_{i}^{ \pm}\right) \neq \vec{f}_{i}^{ \pm}, \quad d g_{j}\left(\vec{f}_{j}^{ \pm}\right) \neq \vec{f}_{j}^{ \pm}
$$

span $T_{x_{i}}\left(\mu F^{\prime}\right)$ and $T_{x_{j}}\left(\mu F^{\prime \prime}\right)$ respectively. According to the Assertion 3 of Proposition 5.3 we have:

$$
d g_{i}\left(\vec{f}_{i}^{ \pm}\right) \perp d g_{j}\left(\vec{f}_{j}^{ \pm}\right)
$$


(Recall that here the orthogonality is defined modulo the parallel translation along curves in $\operatorname{Span}(F)$.) Since both $\operatorname{Span}\left(\mu F^{\prime}\right), \operatorname{Span}\left(\mu F^{\prime \prime}\right)$ intersect $\operatorname{Span}(F)$ orthogonally, the geodesic segment $\overline{x_{i} x_{j}} \subset \operatorname{Span}(F)$ is orthogonal to both $\operatorname{Span}\left(\mu F^{\prime}\right)$ and $\operatorname{Span}\left(\mu F^{\prime \prime}\right)$. Therefore $\mu\left(F^{\prime}\right) \perp \mu\left(F^{\prime \prime}\right)$.

\section{The odd case}

In this section we will construct examples of negatively curved right-angled polygons of groups and their actions on $\mathbb{H}^{p}$ in the case of the odd number of sides.

We define the following polygon of groups. Suppose that we are given finite groups $\Gamma_{1}, \ldots, \Gamma_{n}$. Let $F$ be an $n$-gon $(n \geq 3)$. Below the indices $i$ are taken modulo $n$. We assign the group $G_{x_{i}}=\Gamma_{i} \times \Gamma_{i+1}$ to each vertex $x_{i}$ of $F$. We label each edge $e_{i}$ of $F$ by the group $\Gamma_{i+1}$. The homomorphisms $\Gamma_{i+1} \rightarrow \Gamma_{i} \times \Gamma_{i+1}, \Gamma_{i+1} \rightarrow \Gamma_{i+1} \times \Gamma_{i+2}$ are the natural isomorphisms to the second and the first factor respectively. We set $G_{F}:=\{1\}$. In what follows, let $\mathcal{P}$ denote the resulting polygon of groups (see Figure 9 ) and set $G:=\pi_{1}(\mathcal{P})$.

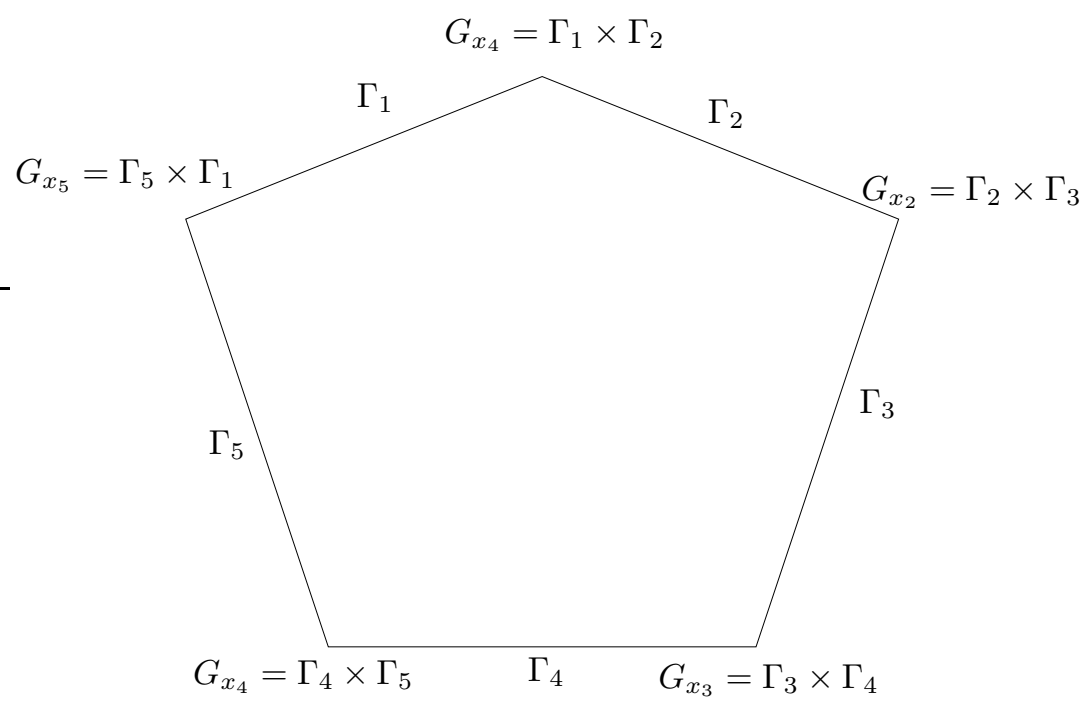

Figure 9

Remark 6.1 Note that $G$ is isomorphic to the cyclic graph-product of the groups $\Gamma_{i}, i=1, \ldots, n$ (see [7] for detailed definition). Indeed, the group $G$ is 
generated by the elements of $\Gamma_{1}, \ldots, \Gamma_{n}$ subject to the relations:

$$
\left[g_{i}, h_{i+1}\right]=1, \forall g_{i} \in \Gamma_{i}, h_{i+1} \in \Gamma_{i+1} .
$$

The polygon of groups $\mathcal{P}$ is negatively curved provided that $n \geq 5$ : For each vertex $x=x_{i} \in \mathcal{P}$, the link of $x$ in the universal cover $X$ of $\mathcal{P}$ is the complete bipartite graph $K_{t_{i-1}, t_{i}}$, where $t_{j}:=\left|G_{e_{j}}\right|, j=1, \ldots, n$.

Theorem 6.2 Suppose that $n \geq 5$, and the polygon of groups $\mathcal{P}$ is as above. Then $G$ admits a properly discontinuous convex-cocompact action on $\mathbb{H}^{p}$ for certain $p=p(\mathcal{P})$.

Proof We will assume that $n=2 k-1$ is odd. Let $e_{1}, \ldots, e_{n}$ denote the edges of the polygon $\mathcal{P}$. First, we construct a homomorphism $\rho: G \rightarrow \operatorname{Isom}\left(\mathbb{H}^{p}\right)$ for some $p$, which is faithful on each vertex group. Let $T$ denote the disjoint union

$$
\bigcup_{i=1}^{n} L k_{e_{i}}
$$

where the link of $e_{i}$ is taken in $X$. One can think of this set as the set of all flags: $(e, f)$, where $e$ is an edge in $F$ and $f$ is a face of $X$ containing $e$. Each group $G_{e_{i}}$ acts naturally on $L k_{e_{i}}$ (since $G_{e_{i}}$ fixes the edge $e_{i} \subset X$ ). We extend this action to the trivial action on the rest of $T$. Thus we get an action

$$
\prod_{i=1}^{n} G_{e_{i}} \curvearrowright T .
$$

Observe that there is a tautological epimorphism

$$
G \rightarrow \prod_{i=1}^{n} G_{e_{i}}
$$

which sends each subgroup $G_{e_{i}} \subset G$ to the subgroup $G_{e_{i}}$ of the direct product. Hence $G$ acts on $T$ through the quotient group $\prod_{i=1}^{n} G_{e_{i}}$.

Let $W$ denote the Euclidean vector space $V$ ect $(T)$ with the orthonormal basis $T$, and set $p:=\operatorname{dim}(W)$, ie,

$$
p=t_{1}+\ldots+t_{n} .
$$

The set $T$ contains distinguished elements $f_{1}, \ldots, f_{n}$ consisting of the flags $\left(e_{i}, F\right)$. The dihedral group $D_{n}$ acts on $F$ and therefore on $\left\{f_{1}, \ldots, f_{n}\right\}$. We extend this action to the rest of $T$ (and hence to $W$ ) by the identity on $T \backslash$ $\left\{f_{1}, \ldots, f_{n}\right\}$. The reflections $R_{j} \in D$ yield isometric involutions $I_{j}$ of $W$. Note 
that (since $n=2 k-1$ is odd) the involution $R_{i+k}$ fixes $x_{i+1}$, hence $I_{i+k}$ permutes the vectors $f_{i}, f_{i+1} \in W$.

Embed the polygon $F$ to $\mathbb{H}^{2}$ as a right-angled regular polygon $\mu(F) \subset \mathbb{H}^{2}$ as in the proof of Theorem 1.1. As before, we will identify $F$ and $\mu(F)$. We embed $\mathbb{H}^{2}$ into $\mathbb{H}^{p}$ as a totally-geodesic subspace. Let $m_{i}$ denote the midpoint of the edge $e_{i} \subset F$. The tangent space $W_{i}:=T_{m_{i}} \mathbb{H}^{p}$ contains a distinguished vector $\overrightarrow{f_{i}}$ which is the unit vector orthogonal to $e_{i}$ and directed inward $F$. Note that this vector is parallel (under the parallel transport along $e_{i}$ ) to the vectors $\vec{f}_{i}^{+}, \vec{f}_{i+1}$ used in the proof of Theorem 1.1 Pick an arbitrary linear isometry $\psi_{1}: W \rightarrow W_{1}$ which sends $f_{1}$ to $\overrightarrow{f_{1}}$. By conjugating via $\psi_{1}$ we transport the linear action $G \curvearrowright W$ to a linear action $G \curvearrowright W_{1}$, exponentiating the latter action we get an isometric action $\rho_{1}: G \curvearrowright \mathbb{H}^{p}$.

We now proceed analogously to the proof of Theorem 1.1? Define linear maps $\psi_{i}: W \rightarrow W_{i}$ so that we have a commutative diagram:

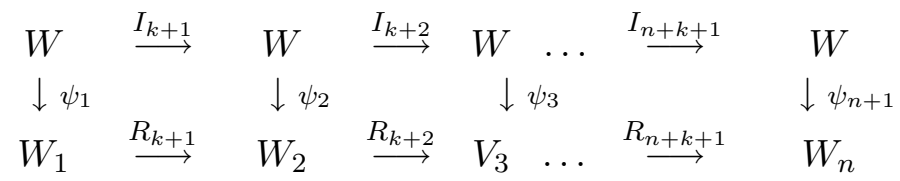

Note that $\psi_{i}\left(f_{i}\right)=\overrightarrow{f_{i}}$ for all $i$. Indeed, $\psi_{1}\left(f_{1}\right)=\overrightarrow{f_{1}}$ by construction. Suppose that $\psi_{i}\left(f_{i}\right)=\overrightarrow{f_{i}}$. Then

$$
\psi_{i+1}\left(f_{i+1}\right)=R_{j} \circ \psi_{i} \circ I_{j}\left(f_{i+1}\right)=R_{j} \circ \psi_{i}\left(f_{i}\right)=R_{j}\left(\overrightarrow{f_{i}}\right)=\overrightarrow{f_{i+1}}
$$

where $j=i+k$.

Observe that $\psi_{n+1} \neq \psi_{1}$. However, $I_{n+k+1} \circ \ldots \circ I_{k+1}=I_{1}$ commutes with $G_{e_{1}} \curvearrowright W$ and $R_{n+k+1} \circ \ldots \circ R_{k+1}=R_{1}$ commutes with $\rho_{1}\left(G_{e_{1}}\right) \curvearrowright \mathbb{H}^{p}$. Hence

$$
\rho_{1}=\rho_{n+1}: G_{e_{1}} \rightarrow \operatorname{Isom}\left(\mathbb{H}^{p}\right) .
$$

It remains to verify that for each $i$ the groups $\rho_{i}\left(G_{e_{i}}\right), \rho_{i+1}\left(G_{e_{i+1}}\right)$ commute. It is elementary to verify that for all $g \in G_{e_{i}}, g^{\prime} \in G_{e_{i+1}}$ the vectors $\psi_{i}\left(g\left(f_{i}\right)\right)$ and $\psi_{i+1}\left(g\left(f_{i+1}\right)\right)$ are mutually orthogonal (after being translated to $T_{x_{i+1}} \mathbb{H}^{p}$ along $\left.e_{i}, e_{i+1}\right)$. The group action $\rho_{i}\left(G_{e_{i}}\right) \curvearrowright T_{x_{i+1}} \mathbb{H}^{p}$ permutes the vectors

$$
\left\{\psi_{i}\left(g\left(f_{i}\right)\right), g \in G_{e_{i}}\right\}
$$

and fixes the orthogonal complement to these vectors; same is true for the action of $G_{e_{i+1}}$ and the vectors

$$
\left\{\psi_{i+1}\left(g^{\prime}\left(f_{i+1}\right)\right), g^{\prime} \in G_{e_{i+1}}\right\}
$$


Hence the groups $\rho_{i}\left(G_{e_{i}}\right), \rho_{i+1}\left(G_{e_{i+1}}\right)$ commute. Therefore we have constructed a homomorphism $\rho: G \rightarrow \operatorname{Isom}\left(\mathbb{H}^{p}\right), \rho\left|G_{e_{i}}=\rho_{i}\right| G_{e_{i}}$.

This action has the same "orthogonality" properties as the homomorphism $\rho$ in the proof of Theorem 1.1, ie, if $i \neq j$ then for all $g \in G_{e_{i}} \backslash\{1\}, g^{\prime} \in G_{e_{j}} \backslash$ $\{1\}$, the hyperbolic planes $\mathbb{H}^{2}, \rho(g) \mathbb{H}^{2}$ and $\rho\left(g^{\prime}\right) \mathbb{H}^{2}$ are mutually orthogonal. Thus, the arguments of the second part of the proof of Theorem [1.1 still work and, by applying Theorem 4.1. we conclude that $\rho$ is discrete, faithful, convexcocompact.

Suppose now that $X$ is a (locally finite) right-angled 2-dimensional hyperbolic building whose fundamental chamber $F$ has $n \geq 6$ vertices. Recall that $X$ is uniquely determined by the thickness $t_{i}$ of the edges $e_{i}$ of $F$, ie, the number of 2 -faces in $X$ containing $e_{i}$. Thus every such building is the universal cover of an $n$-gon $\mathcal{P}$ of finite groups corresponding to a cyclic graph-product. Thickness of the edge $e_{i}$ is the order of the edge group $G_{e_{i}}$ in $\mathcal{P}$.

According to a recent theorem of F. Haglund, [13, all uniform lattices in the building $X$ are commensurable. Hence, as an application of Theorem 6.2 we obtain

Corollary 6.3 Let $H$ be a group acting discretely, cocompactly and isometrically on $X$. Then $H$ contains a finite index subgroup which admits a properly discontinuous convex-cocompact action on $\mathbb{H}^{p}$ for some $p=p(X)$.

\section{Extension of discrete representations}

In this section we discuss the following question:

Suppose that $G \curvearrowright \mathbb{H}^{n}$ is a properly discontinuous isometric action. Is it true that $G$ is isomorphic to a Kleinian group?

Note that the kernel $F$ of the action $G \curvearrowright \mathbb{H}^{n}$ is necessarily finite, therefore we have a short exact sequence

$$
1 \rightarrow F \rightarrow G \rightarrow \bar{G} \rightarrow 1,
$$

where $\bar{G}$ is Kleinian. What we are interested in is whether the group $G$ is itself isomorphic to a Kleinian group. Of course, a necessary condition for this is that $G$ is residually finite. Finding a non-residually finite extension $G$ of a Kleinian group $\bar{G}$ is a very difficult task, and presently such extensions are not known. Nevertheless we have: 
Theorem 7.1 Suppose that $G$ is a residually finite group which fits into a short exact sequence

$$
1 \rightarrow F \rightarrow G \rightarrow \bar{G} \rightarrow 1,
$$

where $\bar{G}$ admits a discrete and faithful representation $\bar{\rho}$ into $\operatorname{Isom}\left(\mathbb{H}^{n}\right)$. Then $G$ also admits a discrete and faithful representation $\rho$ into $\operatorname{Isom}\left(\mathbb{H}^{m}\right)$ for some $m$. Moreover, if $\bar{\rho}$ is convex-cocompact (resp. geometrically finite) then $\rho$ can be taken convex-cocompact (resp. geometrically finite).

Proof The proof of this theorem is modeled on the proof of the well-known fact that a finite extension of a residually finite linear group is again linear, but we present it here for the sake of completeness.

We first lift $\bar{\rho}$ to a homomorphism $\bar{\rho}: G \rightarrow \operatorname{Isom}\left(\mathbb{H}^{n}\right)$, so that $\operatorname{Ker}(\bar{\rho})=F$. Since $G$ is residually finite, there exists a homomorphism

$$
\phi: G \rightarrow Q
$$

where $Q$ is a finite group, so that $\phi \mid F$ is injective. Embed $Q$ in $S O(k)$ for some $k$. The product group $\operatorname{Isom}\left(\mathbb{H}^{n}\right) \times S O(k)$ embeds in $\operatorname{Isom}\left(\mathbb{H}^{n+k}\right)$ as the stabilizer of $\mathbb{H}^{n}$ embedded in $\mathbb{H}^{n+k}$ as a totally-geodesic subspace. Therefore, for $m=n+k$ we get a homomorphism

$$
\rho: G \rightarrow \operatorname{Isom}\left(\mathbb{H}^{n}\right) \times S O(k) \subset \operatorname{Isom}\left(\mathbb{H}^{m}\right)
$$

given by

$$
\rho(g)=(\bar{\rho}(g), \phi(g)) .
$$

It is clear that $\rho(g) \mid \mathbb{H}^{n}=\bar{\rho}(g)$ and therefore $\rho$ is faithful and $\rho(G) \subset \operatorname{Isom}\left(\mathbb{H}^{m}\right)$ is discrete. Moreover, $\Lambda(\rho(G))=\Lambda(\bar{\rho}(\bar{G}))$. Recall that geometrically finite and convex-cocompact actions can be detected by considering the dynamics of a discrete group on its limit set (see [5]). Therefore, if $\bar{\rho}$ is convex-cocompact (resp. geometrically finite) then $\rho$ is also convex-cocompact (resp. geometrically finite).

Combining Theorem [7.1 with Theorem 5.1 we get Theorem 1.1

\section{Example of a nonlinear Gromov-hyperbolic group}

Theorem 8.1 There exists an infinite hyperbolic group $G$ such that each representation of $G$ to $G L(m, \mathbf{k})$ factors through a finite group. In particular, $G$ is nonlinear. Here $\mathbf{k}$ is an abritrary field. 
Proof Let $\Gamma$ be a uniform lattice in a quaternionic hyperbolic space $\mathbb{H}^{n}, n \geq$ 2. Since $\mathbb{H} \mathbb{H}^{n}$ is negatively curved, the group $\Gamma$ is hyperbolic. Clearly, the group $\Gamma$ is a nonelementary hyperbolic group; hence $\Gamma$ admits an infinite proper quotient $\Gamma \rightarrow G$ where $G$ is a hyperbolic group (see [1] or [16]). We first consider the case when $\mathbf{k}$ has zero characteristic. Then without loss of generality we can assume that we are given a linear representation $\rho: G \rightarrow G L(N, \mathbb{R})$. We will show that $\rho(G)$ is finite by using the standard "adelic" trick. The reader can find similar applications of this argument in Margulis' proof of arithmeticity of higher rank lattices (see [19, 25]), and in Tits' proof of the Tits alternative, [23.

The representation $\rho$ lifts to a linear representation $\widetilde{\rho}: \Gamma \rightarrow G L(N, \mathbb{R})$. Let $L$ denote the Zariski closure of $\widetilde{\rho}(\Gamma)$ in $G L(N, \mathbb{R})$. Let $S$ denote the solvable radical of $L$. We first consider the case when $L^{\prime}:=L / S$ is a reductive group with nontrivial noncompact factor $H$. Then the projection $\Gamma \rightarrow G \rightarrow L \rightarrow H$ has Zariski dense image. Hence, according to Corlette's Archimedean superrigidity theorem 9], the representation $\Gamma \rightarrow H$ extends to $\operatorname{Isom}\left(\mathbb{H} \mathbb{H}^{n}\right)$. This however contradicts the assumption that the projection $\Gamma \rightarrow G$ is not $1-1$. Therefore the group $L^{\prime}$ is a compact algebraic group.

Suppose that the projection $\rho(G) \subset L^{\prime}$ is infinite. As a compact Lie group, $L^{\prime}$ is isomorphic to a subgroup of $O(M)$. Since $G$ satisfies property (T),

$$
H^{1}\left(G, o(M)_{A d(\rho)}\right)=0,
$$

where $o(M)$ is the Lie algebra of $O(M)$. Vanishing of the above cohomology group implies that the space $\operatorname{Hom}(G, O(M)) / O(M)$ is finite. Hence, analogously to the proof of Theorem 7.67 in 21], $\rho$ is conjugate to a representation $\rho^{\prime}: G \rightarrow O(M)$ for which

$$
\rho^{\prime}(G) \subset K(F) \subset O(M, F) \subset G L(M, F),
$$

where $F$ is a number field and $K(\mathbb{R})$ is the Zariski closure of $\rho^{\prime}(G)$.

One would like to replace the representation $\rho$ with another representation $\phi$ of the group $G$, whose image is Zariski dense in a certain noncompact algebraic group and so that $\operatorname{Ker}(\phi)=\operatorname{Ker}(\rho)$. The most obvious thing to try is to find an element $\sigma$ of the Galois group $\operatorname{Gal}(\mathbb{C} / \mathbb{Q})$, so that the image of $\phi=\sigma(\rho)$ is not relatively compact. This does not necessarily work. Note however, that the restriction of the norm on $\mathbb{C}$ to $\sigma(F)$ gives rise to an Archimedean valuation on $F$. The idea of the adelic trick is to use non-Archimedean valuations $v$ of $F$ together with Archimedean ones. This is done by introducing the ring of adeles of $F$, which is a certain subset of the product

$$
\prod_{v \in \operatorname{Val}(F)} F_{v}
$$


where $F_{v}$ is the completion of $F$ with respect to the valuation $v$.

Let $\mathbb{A}(F)$ denote the ring of adeles of $F$; then the diagonal embedding $F \hookrightarrow$ $\mathbb{A}(F)$ has discrete image (see for example [18]). Hence the diagonal embedding

$$
\rho^{\prime}(G) \hookrightarrow G L(M, \mathbb{A}(F))
$$

also has discrete image. If the projection of $\rho^{\prime}(G)$ to each factor $G L\left(M, F_{v}\right)$ were relatively compact, the image of $\rho^{\prime}(G)$ in $G L(M, \mathbb{A}(F))$ would be compact as well. However a discrete subset of a compact is finite, which contradicts the assumption that $\rho(G)$ is infinite.

Thus there exists a valuation $v$ of $F$ so that the image of the projection

$$
\rho^{\prime}(G) \rightarrow K\left(F_{v}\right) \subset G L\left(M, F_{v}\right)
$$

is not relatively compact. In case when $v$ is an Archimedean valuation, we can again apply Corlette's Archimedean superrigidity [9] to get a contradiction. Hence such $v$ has to be nonarchimedean. Therefore the representation $\Gamma \rightarrow \rho^{\prime}(G) \rightarrow G L\left(M, F_{v}\right)$ corresponds to an isometric action of $\Gamma$ on a locally finite Euclidean building $X$. However, by the non-Archimedean superrigidity theorem of Gromov and Schoen [12, $\Gamma$ fixes a point in $X$. Therefore the image of $\rho^{\prime}(G)$ in $G L\left(M, F_{v}\right)$ is relatively compact, which is a contradiction. Hence $\rho^{\prime}(G)$ is finite. It follows that the group $L$ is commensurable to its solvable radical $S$; hence $\rho(G)$ is a virtually solvable group. By applying property (T) again, we conclude that $\rho(G)$ is finite.

We now consider the case when $\mathbf{k}$ has positive characteristic; since the argument is similar to the zero characteristic case, we give only a sketch. Under the above assumptions, $\rho(G) \subset G L(m, F)$, where $F$ is a finitely generated field (of positive characteristic). The field $F$ is an extension

$$
F_{q} \subset E \subset F
$$

where $F_{q}$ is a finite field, $F_{q} \subset E$ is an purely transcendental extension and $E \subset F$ is an algebraic extension (see [15, Chapter VI.1]). Since $F$ is finitely generated, $F / E$ is finite-dimensional and therefore, by passing to a bigger matrix group, we reduce the problem to the case when $F=E$ is a purely transcendental extension, which necessarily has finite transcendence degree. Therefore we reduced to the case of $\rho: G \rightarrow G L(N, F)$, where $F=F_{q}\left(t_{1}, \ldots, t_{m}\right)$ is the field of rational functions with coefficients in $F_{q}$.

Then we associate with each variable $t_{j}^{ \pm 1}$ a discrete valuation $v_{ \pm j}$ and an action $G \curvearrowright X_{ \pm j}$ on the corresponding Euclidean building. The non-Archimedean superrigidity theorem of Gromov and Schoen [12] shows that for each $\pm j$ the 
action $G \curvearrowright X_{ \pm j}$ has a fixed point. Therefore the matrix coefficients of $\rho(G)$ have bounded degree with respect to all the variables $t_{1}^{ \pm 1}, \ldots, t_{m}^{ \pm 1}$. Hence, since $F_{q}$ is finite, the matrix coefficients of $\rho(G)$ belongs to a finite subset of $F$ and thus $\rho(G)$ is finite.

Remark 8.2 After this paper was written, I was informed by Alain Valette that he also knew how to prove Theorem 8.1. I am sure that other people were also aware of this proof since all the arguments here are quite standard.

\section{References}

[1] W Ballmann, J Światkowski, On $L^{2}$-cohomology and property (T) for automorphism groups of polyhedral cell complexes, Geom. Funct. Anal. 7 (1997) 615-645 MathReview

[2] A F Beardon, The geometry of discrete groups, Graduate Texts in Mathematics 91, Springer-Verlag, New York (1983) MathReview

[3] M Bonk, O Schramm, Embeddings of Gromov hyperbolic spaces, Geom. Funct. Anal. 10 (2000) 266-306 MathReview

[4] M Bourdon, Sur la dimension de Hausdorff de l'ensemble limite d'une famille de sous-groupes convexes co-compacts, C. R. Acad. Sci. Paris Sér. I Math. 325 (1997) 1097-1100 MathReview

[5] B H Bowditch, Geometrical finiteness for hyperbolic groups, J. Funct. Anal. 113 (1993) 245-317 MathReview

[6] B H Bowditch, Geometrical finiteness with variable negative curvature, Duke Math. J. 77 (1995) 229-274 MathReview

[7] MR Bridson, A Haefliger, Metric spaces of non-positive curvature, Grundlehren series 319, Springer-Verlag, Berlin (1999) MathReview

[8] J A Carlson, D Toledo, Harmonic mappings of Kähler manifolds to locally symmetric spaces, Inst. Hautes Études Sci. Publ. Math. (1989) 173-201 MathReview

[9] K Corlette, Archimedean superrigidity and hyperbolic geometry, Ann. of Math. (2) 135 (1992) 165-182 MathReview

[10] A Felikson, P Tumarkin, A series of word-hyperbolic Coxeter groups, arXiv:math.GR/0507389

[11] M Gromov, Hyperbolic groups, from: "Essays in group theory", Math. Sci. Res. Inst. Publ. 8, Springer, New York (1987) 75-263 MathReview

[12] M Gromov, R Schoen, Harmonic maps into singular spaces and p-adic superrigidity for lattices in groups of rank one, Inst. Hautes Études Sci. Publ. Math. (1992) 165-246 MathReview 
[13] F Haglund, Commensurabilité de résaux uniformes dans les immeubles à angles droits, preprint (2003)

[14] F Haglund, D Wise, in preparation

[15] T W Hungerford, Algebra, Graduate Texts in Mathematics 73, SpringerVerlag, New York (1980) MathReview

[16] S V Ivanov, A Y Ol'shanskiı,, Hyperbolic groups and their quotients of bounded exponents, Trans. Amer. Math. Soc. 348 (1996) 2091-2138 MathReview

[17] M Kapovich, Hyperbolic manifolds and discrete groups, Progress in Mathematics 183, Birkhäuser Boston Inc. Boston, MA (2001) MathReview

[18] S Lang, Algebraic numbers, Addison-Wesley Publishing Co. Inc. Reading, Mass.-Palo Alto-London (1964) MathReview

[19] G A Margulis, Discrete subgroups of semisimple Lie groups, Ergebnisse series 17, Springer-Verlag, Berlin (1991) MathReview

[20] G D Mostow, Y T Siu, A compact Kähler surface of negative curvature not covered by the ball, Ann. of Math. (2) 112 (1980) 321-360 MathReview

[21] MS Raghunathan, Discrete subgroups of Lie groups, Springer-Verlag, New York (1972) MathReview

[22] J Światkowski, A class of automorphism groups of polygonal complexes, Q. J. Math. 52 (2001) 231-247 MathReview

[23] J Tits, Free subgroups in linear groups, J. Algebra 20 (1972) 250-270 MathReview

[24] D T Wise, The residual finiteness of negatively curved polygons of finite groups, Invent. Math. 149 (2002) 579-617 MathReview

[25] R J Zimmer, Ergodic theory and semisimple groups, Monographs in Mathematics 81, Birkhäuser Verlag, Basel (1984) MathReview 